\title{
The propagation of a liquid bolus along a liquid-lined flexible tube
}

\author{
By P. D. HOWELL ${ }^{1} \dagger$, S. L. WATERS ${ }^{2} \ddagger$ \\ AND J. B. GROTBERG ${ }^{2}$ \\ ${ }^{1}$ Mathematical Institute, Oxford University, 24-29 St Giles, Oxford, OX1 3LB, UK \\ ${ }^{2}$ Biomedical Engineering Department, University of Michigan, 3304 G G Brown, 2350 Hayward, \\ Ann Arbor, MI 48109, USA.
}

(Received 26 September 1996 and in revised form 17 September 1999)

We use lubrication theory and matched asymptotic expansions to model the quasisteady propagation of a liquid plug or bolus through an elastic tube. In the limit of small capillary number, asymptotic expressions are found for the pressure drop across the bolus and the thickness of the liquid film left behind, as functions of the capillary number, the thickness of the liquid lining ahead of the bolus and the elastic characteristics of the tube wall. These results generalize the well-known theory for the low capillary number motion of a bubble through a rigid tube (Bretherton 1961). As in that theory, both the pressure drop across the bolus and the thickness of the film it leaves behind vary like the two-thirds power of the capillary number. In our generalized theory, the coefficients in the power laws depend on the elastic properties of the tube.

For a given thickness of the liquid lining ahead of the bolus, we identify a critical imposed pressure drop above which the bolus will eventually rupture, and hence the tube will reopen. We find that generically a tube with smaller hoop tension or smaller longitudinal tension is easier to reopen. This flow regime is fundamental to reopening of pulmonary airways, which may become plugged through disease or by instilled/aspirated fluids.

\section{Introduction}

A pulmonary airway may be described as a compliant tube with a liquid lining. It is well known that this lining is susceptible to a Rayleigh instability driven by capillarity at its free surface. If the volume of liquid present is sufficiently large, the instability culminates in the formation of a liquid bridge or plug that blocks the flow of air along the airway (Hughes, Rosenzweig \& Kivitz 1970; Macklem, Proctor \& Hogg 1970; Kamm \& Schroter 1989; Halpern \& Grotberg 1992, 1993; Otis et al. 1993). This phenomenon, known as airway closure, occurs naturally in healthy adults towards the end of expiration. However, respiratory problems may ensue if the airways remain closed for a significant proportion of the breathing cycle. Mechanisms which can increase the likelihood and/or duration of airway closure include increased liquid content in the lung, increased airway compliance and increased surface tension at the

$\dagger$ Present address: School of Mathematical Sciences, University of Nottingham, University Park, Nottingham, NG7 2RD, UK.

$\ddagger$ Present address: Department of Applied Mathematics and Theoretical Physics, University of Cambridge, Silver Street, Cambridge, CB3 9EW, UK. 
air-liquid interface. For example, premature infants frequently suffer from Respiratory Distress Syndrome, caused by the increased surface tension resulting from a deficiency in pulmonary surfactant, while emphysema and asthma can both adversely affect both airway wall compliance and airway lining thickness. Also, airway closure tends to occur in the lower regions of the lung due to gravitational effects which compress the lung there. In microgravity environments, airway closure has been shown to exist (Prisk et al. 1995) but is likely to be more homogeneously distributed, leaving fewer lung segments available for gas exchange during a portion of the respiratory cycle.

Once a liquid bolus forms, it propagates along the airway under the pressure drops imposed across it during respiration. Reopening of the airway occurs if and when the bolus volume decreases to the point that it ruptures. In this paper we model the low capillary number propagation of a liquid bridge along an elastic tube under an imposed pressure drop. Our aim is to determine criteria for airway reopening and to ascertain how these depend on such physiological parameters as surface tension, airway lining thickness and airway wall elastic properties.

The capillary instability of a cylinder of viscous liquid was first identified by Rayleigh $(1879,1892)$. The corresponding result for a liquid annulus was derived by Goren (1962), and a weakly nonlinear analysis was carried out by Hammond (1983). With the aim of modelling airway closure, fully nonlinear numerical analyses were performed by Johnson et al. (1991) and Otis et al. (1993), the latter including insoluble surfactant, and flexibility of the tube walls was incorporated by Halpern \& Grotberg (1992, 1993).

Pioneering analysis of the low capillary number propagation of a bubble along a capillary tube was performed by Bretherton (1961), and recast in the terminology of matched asymptotic expansions by Park \& Homsy (1984), see also Schwartz, Princen \& Kiss (1986), Wong, Radke \& Morris $(1995 a, b)$. Their results may equally well be applied to the propagation of a bolus as to that of a bubble. Analogous techniques have been widely applied, for example, to coating flows and gravity draining (Landau \& Levich 1942; Ruschak \& Scriven 1977; Wilson 1982).

The propagation of a semi-infinite bubble into a liquid-filled elastic channel was considered by Gaver et al. (1996), as a simple model of airway reopening. By employing boundary integral methods in a region near the bubble nose and patching to lubrication solutions ahead of and behind the nose, they extended their theory to higher capillary numbers than those admitted by the theory of Bretherton (1961). In the low capillary number limit, their theory agrees with that of $\S 3$ in this paper.

The model we use for the elastic response of the wall was originally derived by Atabek \& Lew (1966) and used by Halpern \& Grotberg (1992) in modelling airway closure. The tube wall is characterized by a constant longitudinal tension $T_{l}$ and a circumferential tension that is constituted in terms of its Young's modulus, $E$, thickness, $d$, and Poisson's ratio, $v$. The following dimensionless parameters then define the tube's elastic properties:

$$
\Gamma=\frac{\sigma\left(1-v^{2}\right)}{E d}, \quad T=\frac{T_{l}}{\sigma},
$$

which are the dimensionless compliance and tension respectively. In this paper, we shall consider the circumferential stress to be much greater than the constant fluid surface tension $\sigma$. Hence the dimensionless wall compliance is small and the wall is relatively stiff. We shall consider the longitudinal wall tension to be much less than the circumferential stress, corresponding to $T_{l} \ll E d$, so that $T \Gamma \ll 1$. We consider two limiting cases for the relative sizes of $T_{l}$ and $\sigma$, i.e. for the size of $T$ : (i) 


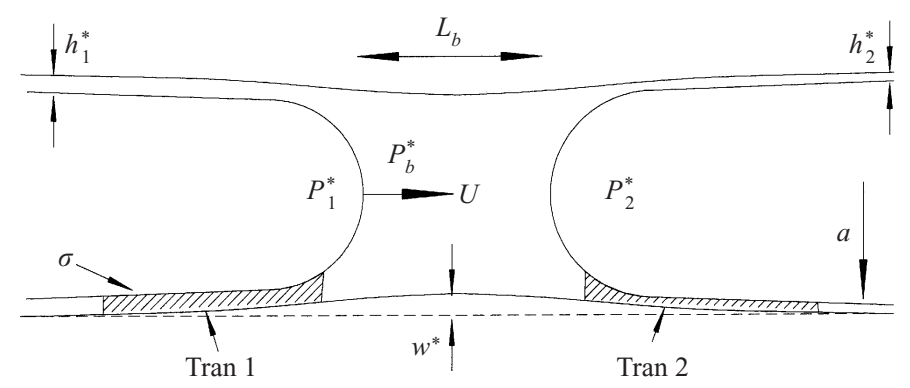

FIGURE 1. Definition sketch of a propagating liquid bolus.

longitudinal tension, $T$, much less than 1 - referred to as the weak tension case; and (ii) longitudinal tension significantly larger than the wall compliance - referred to as the tension dominated case.

In $\S 2$ we formulate the problem and give the dimensionless governing equations and boundary conditions. In $\S 3$ we derive a simple model for the propagation of a liquid bolus along a flexible tube for the weak tension case. The dimensionless wall compliance is assumed to be sufficiently small that the wall displacements are of the same order as the thickness of the liquid lining. In this limit we deduce a critical pressure drop which must be imposed across the bolus if the thickness of the film it leaves behind is to exceed that of the film ahead of it. This gives a minimum criterion for eventual rupture of the bolus and reopening of the tube. In $\S 4$ we relax the restrictions on the wall compliance imposed in $\S 3$, and derive a theory for bolus propagation through a relatively compliant tube in the tension dominated case. Again, minimum criteria for tube reopening are identified. In $\S 5$, we draw conclusions and discuss the physiological significance of our results.

\section{Formulation}

\subsection{Model description}

Figure 1 gives a schematic description of the bolus model. The bolus propagates in the laboratory frame at constant speed $U$ under the pressure drop imposed across it. The equilibrium radius of the tube, corresponding to a (dimensionless) transmural pressure difference of 1 , is $a$. The length of the liquid bolus is $L_{b}$ which we assume to be $O(a)$. Inertia is neglected throughout the paper, so the only relevant fluid parameters are its viscosity $\mu$ and density $\rho$. The fluid has constant surface tension $\sigma$.

Throughout this paper we shall use superscript $*$ to denote dimensional variables. We use cylindrical polar coordinates $\left(r^{*}, \theta, z^{*}\right)$ fixed to the bolus, with the $z^{*}$-coordinate the direction of the bolus motion in the laboratory frame. The velocity components in this frame are $\boldsymbol{u}^{*}=\left(u_{r}^{*}, 0, u_{z}^{*}\right)$ and $P^{*}$ is the fluid pressure. The air pressures behind and ahead of the bolus are $P_{1}^{*}, P_{2}^{*}$ respectively. We seek steady-state solutions in the frame travelling with the bolus.

The inward tube displacement is denoted by $w^{*}\left(z^{*}, t^{*}\right)$ and the thickness of the liquid film by $h^{*}\left(z^{*}, t^{*}\right)$. It is also useful to define the free-surface displacement $\eta^{*}=w^{*}+h^{*}$. Thus the positions of the liquid-wall and the air-liquid interfaces are given in cylindrical polar coordinates by $r^{*}=a-w^{*}\left(z^{*}, t^{*}\right)$ and $r^{*}=a-\eta^{*}\left(z^{*}, t^{*}\right)$ respectively. Ahead of the bolus, the tube has a 'precursor' liquid lining thickness $h_{2}^{*}$. The bolus leaves behind a 'trailing film' of thickness $h_{1}^{*}$. 


\subsection{Dimensionless governing equations and parameters}

In order to determine the dimensionless parameters that describe the relative importance of the various features of the problem, we non-dimensionalize as follows:

$$
\left(r^{*}, z^{*}, h^{*}, w^{*}\right)=a(r, z, h, w), \quad \boldsymbol{u}^{*}=U \boldsymbol{u}, \quad P^{*}=\frac{\sigma}{a} P .
$$

We assume slow viscous flow $(\rho U a / \mu \ll 1)$, so that the governing equations are Stokes equations and continuity. Non-dimensionalizing according to (2.1) the dimensionless equations are

$$
\nabla P=\frac{1}{3} C a \nabla^{2} \boldsymbol{u}, \quad \nabla \cdot \boldsymbol{u}=0,
$$

where $C a=3 \mu U / \sigma$ is the capillary number, and is the ratio of viscous to capillary effects (the factor 3 is included in this definition to simplify future expressions).

The air-liquid and liquid-wall interfaces are free surfaces whose shape is determined by the balance of viscous stress, fluid pressure and wall stress. At the air-liquid interface, the following stress balance holds:

$$
\tau \cdot \hat{\boldsymbol{n}}_{I}=\left(K-P_{1}\right) \hat{\boldsymbol{n}}_{I} \quad \text { at } \quad r=1-\eta,
$$

where $\hat{\boldsymbol{n}}_{I}=\left(n_{r}, 0, n_{z}\right)$ is the unit outward normal to the interface and $\tau=-P \boldsymbol{I}+$ $C a\left(\nabla \boldsymbol{u}+\nabla \boldsymbol{u}^{T}\right)$ is the fluid stress tensor. $K=\nabla_{S} \cdot \hat{\boldsymbol{n}}_{I}$ is the interfacial curvature, where $\nabla_{s}=\left(\boldsymbol{I}-\hat{\boldsymbol{n}}_{I} \hat{\boldsymbol{n}}_{I}\right) \cdot \nabla$ is the surface divergence operator.

The dimensionless form of the wall law (Atabek \& Lew 1966) is

$$
-\frac{1}{\Gamma} \frac{w}{(1-w)^{2} \sqrt{1+w_{z}^{2}}}+T \frac{w_{z z}}{\left(1+w_{z}^{2}\right)^{3 / 2}}=P-P_{e x t} \quad \text { at } \quad r=1-w
$$

where $P_{\text {ext }}$ is the pressure outside the tube, chosen to make $w \rightarrow 0$ far behind the bolus at equilibrium. Far upstream, $P_{1}-P=1$ and so we set $P_{\text {ext }}=P_{1}-1$. If we now let $P=P_{1}+P^{\prime}$, the wall equation is (dropping primes)

$$
-\frac{w}{(1-w)^{2} \sqrt{1+w_{z}^{2}}}+\Gamma T \frac{w_{z z}}{\left(1+w_{z}^{2}\right)^{3 / 2}}=\Gamma(1+P) \quad \text { at } \quad r=1-w .
$$

The remaining boundary conditions are that

$$
\begin{array}{rlll}
\boldsymbol{u} \cdot \hat{\boldsymbol{n}}_{I}=0 & \text { at } & r=(1-\eta), \\
\boldsymbol{u} \cdot \hat{\boldsymbol{n}}_{w}=0 & \text { at } & r=(1-w), \\
(\boldsymbol{u}+\hat{\boldsymbol{z}}) \cdot \hat{\boldsymbol{t}}_{w}=0 & \text { at } & r=(1-w),
\end{array}
$$

where $\hat{\boldsymbol{n}}_{w}$ and $\hat{\boldsymbol{t}}_{w}$ are the unit normal and unit tangent at the liquid-wall interface respectively. Equation (2.6a) corresponds to the kinematic condition that fluid in the interface remains in the interface and $(2.6 b)$ refers to no fluid penetration at the wall. Equation (2.6c) is the no-slip condition at the wall.

We assume that in the laboratory frame, no flow exists at $z= \pm \infty$. In the bolus frame of reference, the dimensionless upstream and downstream conditions are thus

$$
\boldsymbol{u} \rightarrow-\hat{z} \quad \text { as } \quad z \rightarrow \pm \infty .
$$

From (2.5) we deduce that in general $w$ scales with $\Gamma$. Since $\Gamma \ll 1$, the wall displacement is relatively small. If we let $w=\Gamma w^{\prime}$, then neglecting terms of $O\left(\Gamma^{2}\right)$ in (2.5) we obtain the following linear wall law (dropping primes):

$$
-w+\Gamma T w_{z z}=1+P .
$$


In $\S 3$ we consider the propagation of a liquid bolus along a compliant tube in the weak tension limit, corresponding to $T \ll 1$. We also assume that wall compliance, $\Gamma$, is of order $\mathrm{Ca}^{2 / 3}$. The leading-order expression for the pressure drop across the bolus is independent of the longitudinal wall tension. In $\S 4$ we consider the tension dominated limit corresponding to $\Gamma \ll T \ll \Gamma^{-1}$.

As shown by Bretherton (1961), the dimensionless trailing film thickness $h_{1}$ scales with $C a^{2 / 3}$. In $\S 3$ we consider the simple case where the scalings for $w$ and $h$ are the same, i.e. $\Gamma \sim O\left(C a^{2 / 3}\right)$. We find that the length scale over which the wall adjusts to the different pressures exerted on it is $O(\sqrt{\Gamma}) \sim O\left(\mathrm{Ca}^{1 / 3}\right)$, the same length scale over which the free-surface curvature adjusts. In $\S 4$ this restriction on the wall compliance is lifted. In this tension dominated case, we find now that the length scale over which the wall adjusts is $O(\sqrt{\Gamma T})$ which is uncoupled from the $O\left(C a^{1 / 3}\right)$ length scale over which the fluid surface curvature adjusts.

\section{Weak tension case}

In this section we consider a simple model for the propagation of a liquid bolus along a flexible tube in the weak tension limit $T \ll 1$. We also assume that $\Gamma \sim$ $O\left(\mathrm{Ca}^{2 / 3}\right)$. We derive a generalized version of the well-known Landau-Levich equation that includes elastic deflections of the tube wall. The generalized equation admits solutions whose behaviour differs markedly from that of solutions of the LandauLevich equation, though unfortunately detailed analysis of it is beyond the scope of this paper.

\subsection{Quasi-steady solution for the rear meniscus}

Consider a liquid bolus as shown in figure 1. The dimensionless Stokes equations reveal that

$$
\nabla P_{b}=\frac{1}{3} C a \nabla^{2} \boldsymbol{u}
$$

where $P_{b}$ is the (dimensionless) pressure in the bolus. Our analysis is based upon the assumption that the capillary number is small, i.e. that the bolus propagates at relatively low speed. From (3.1),

$$
P_{b} \sim \text { const. }+O(\mathrm{Ca}),
$$

which implies that, up to $O(\mathrm{Ca})$, the two menisci on either side of the bolus must be surfaces of constant mean curvature, that curvature (dimensionless) being $P_{1}-P_{b}$ and $P_{2}-P_{b}$ respectively. We define here the dimensionless pressure drops across the rear and front menisci and across the bolus as

$$
\Delta P_{1}=P_{1}-P_{b}, \quad \Delta P_{2}=P_{2}-P_{b}, \quad \Delta P=P_{1}-P_{2}=\Delta P_{1}-\Delta P_{2},
$$

respectively.

Imposing that the surfaces be axisymmetric and analytic, the only admissible constant mean curvature surfaces are spherical caps. The equation of such a cap is

$$
r^{* 2}=R^{*}\left(z^{*}\right)^{2} \equiv R_{c}^{* 2} \sec ^{2} \theta_{c}-\left(z^{*}+R_{c}^{*} \tan \theta_{c}\right)^{2},
$$

where the cap makes a constant angle $\theta_{c}$ with a tube of radius $R_{c}^{*}$ at $z^{*}=0$. For convenience we assume that $r^{*}, R^{*}, R_{c}^{*}$ and $z^{*}$ are all non-dimensionalized with $a$, and hence

$$
\Delta P_{1}=\frac{\cos \theta_{c}}{R_{c}}(1+O(C a)) .
$$




\subsection{Lubrication analysis of the rear transition region}

A spherical cap as described above cannot be joined analytically to the uniform thickness film ahead of or behind the bolus. Instead there are transition regions (marked 'Tran 1' and 'Tran 2' in figure 1) between the uniform films and the menisci, in which the assumption of constant pressure fails and viscous dissipation becomes important. However, asymptotic simplification can still be achieved since in the transition regions the liquid layer is thin, with a slowly varying free surface, so we employ lubrication theory.

Consider first the rear transition region. We look for a steady state in a frame travelling with the bolus. We assume the transition region to be of length $\hat{L}$ and define a new variable $\zeta$ via

$$
\zeta=z / \hat{L},
$$

where $\hat{L}$ will determined subsequently. The film thickness $h$ and the free-surface displacement $\eta$ are scaled with the dimensionless trailing film thickness $h_{1}$. We define $\varepsilon$ such that $h_{1}=\varepsilon \hat{L}$ and a new variable $y$ such that

$$
r=1-\varepsilon \hat{L} y .
$$

Thus $y=\eta$ corresponds to the air-liquid interface and $y=w$ corresponds to the liquid-wall interface. Note also that $h=\eta-w$.

The fluid pressure at the wall is given by

$$
P \sim-K-\frac{\varepsilon}{\hat{L}} \frac{h R_{\zeta} K_{\zeta}}{1+R_{\zeta}^{2}},
$$

where $R=1-w$. The leading-order momentum equations in the film are

$$
\begin{aligned}
& \frac{\partial P}{\partial \zeta}=\frac{1}{3} \frac{C a}{\hat{L} \varepsilon^{2}} \frac{\partial^{2} u_{z}}{\partial y^{2}}+O\left(\frac{C a}{\varepsilon}, C a \hat{L}, \frac{C a}{\hat{L}}\right), \\
& \frac{\partial P}{\partial y}=O\left(\frac{C a}{\hat{L}}, C a \varepsilon \hat{L}\right) .
\end{aligned}
$$

Note that (3.9) implies that we need to take $\hat{L} \varepsilon^{2}=O(C a)$ and hence, given the already made assumption that $\varepsilon \hat{L} \ll 1$, the terms $O(C a / \varepsilon, C a \hat{L}, C a / \hat{L})$ are much less than 1 .

The boundary conditions at the liquid-wall interface are (to leading order)

$$
u_{z}=-1, \quad u_{r}=\frac{\partial w}{\partial \zeta} \quad \text { at } \quad y=w,
$$

and the normal and tangential stress conditions at the air-liquid interface reduce to

$$
-h_{\zeta}+\frac{\partial}{\partial \zeta}\left(\frac{\hat{L} \varepsilon^{2}}{C a}\left(\varepsilon \hat{L} \eta_{\zeta}+\frac{\varepsilon}{\hat{L}} \eta_{\zeta \zeta \zeta}\right) h^{3}\right)=0 .
$$

We find that $\varepsilon \sim C a^{1 / 3}, \hat{L} \sim C a^{1 / 3}$ and, applying the upstream boundary condition, the governing equation for the film thickness is

$$
h^{3} \eta_{\zeta \zeta \zeta}=h-1+O\left(\mathrm{Ca}^{2 / 3}\right) .
$$

Rescaling the wall law (2.8) appropriately we obtain the leading-order dimensionless wall law

$$
w=G_{1} \eta_{\zeta \zeta}+O\left(C a^{2 / 3}\right),
$$


where

$$
G_{1}=\Gamma\left(1 / h_{1}\right)^{2} C a^{2 / 3} \sim O\left(\Gamma C a^{-2 / 3}\right)
$$

is our dimensionless flexibility coefficient, which we assume to be an order-one constant throughout this section. More compliant walls, for which $G_{1} \gg 1$ are considered in $\S 4$.

Equations (3.12) and (3.13) combine to a single ordinary differential equation for $\eta$, valid up to $O\left(C a^{2 / 3}\right)$, namely

$$
\eta_{\zeta \zeta \zeta}=\frac{\eta-G_{1} \eta_{\zeta \zeta}-1}{\left(\eta-G_{1} \eta_{\zeta \zeta}\right)^{3}} .
$$

This equation clearly reduces to the Landau-Levich equation as $G_{1} \rightarrow 0$, that is as the tube becomes increasingly rigid.

An initial condition for (3.15) is that the film becomes uniform far away from the bolus. We linearize equation (3.15) by substituting

$$
\eta \sim 1+\varepsilon_{1} \mathrm{e}^{l \zeta} \text { as } \zeta \rightarrow-\infty,
$$

where $\varepsilon_{1} \ll 1$. The eigenvalue $l\left(G_{1}\right)$ satisfies the cubic polynomial

$$
l^{3}+G_{1} l^{2}-1=0,
$$

which has one positive real root and two complex roots with negative real part. To ensure that we approach the uniform film as $\zeta \rightarrow-\infty$, we choose as our initial condition the exponentially decaying eigenfunction. This one initial condition specifies the problem up to an arbitrary translation in $\zeta$.

The solution is found to behave quadratically for large, positive $\zeta$ :

$$
\eta \sim \frac{1}{2} A_{1} \zeta^{2}+B_{1} \zeta+C_{1} \quad \text { as } \quad \zeta \rightarrow \infty,
$$

where $A_{1}, B_{1}$ and $C_{1}$ are numerically determined constants for each given value of $G_{1}$. Notice that these constants are not all uniquely determined because the origin for $\zeta$ may be chosen arbitrarily. However $A_{1}$ and the combination $F_{1}=A_{1} C_{1}-\frac{1}{2} B_{1}^{2}$ are independent of the origin chosen for $\zeta$ and so are determined uniquely for any fixed $G_{1}$.

\subsection{Matching}

The final step is to match the transition region with the outer, capillary static solution of §3.1. Formally, we apply Van Dyke's matching rule (Van Dyke 1964) to the three-term outer solution (in which the free surface is given up to $O(C a)$ by (3.4)) and the one-term inner solution found above. The matching can only be achieved if $h_{1}=\beta C a^{2 / 3}$ where $\beta$ is an order-one constant. Then expanding the equation of the spherical cap, (3.4), about $z=0$ in inner variables $\left(R \sim R^{(0)}+C a^{1 / 3} R^{(1)}+\cdots\right)$ we obtain

$$
\begin{aligned}
R\left(\beta C a^{1 / 3} \zeta\right) \sim & R^{(0)}(0)+C a^{1 / 3}\left(R^{(1)}(0)+\beta \zeta R_{z}^{(0)}(0)\right) \\
& +C a^{2 / 3}\left(R^{(2)}(0)+\beta \zeta R_{z}^{(1)}(0)+\frac{1}{2} \beta^{2} \zeta^{2} R_{z z}^{(0)}(0)\right)+\cdots .
\end{aligned}
$$

Recall that in the transition region, $R=1-C a^{2 / 3} \beta \eta$, where $\eta$ is given asymptotically by (3.18). Hence, matching (3.19) with (3.18) gives

$$
\left.\begin{array}{l}
R^{(0)}(0)=1, \quad R^{(1)}(0)=0, \quad R_{z}^{(0)}(0)=0 \\
R^{(2)}(0)=-\beta C_{1}, \quad R_{z}^{(1)}(0)=-B_{1}, \quad \beta R_{z z}^{(0)}(0)=-A_{1},
\end{array}\right\}
$$




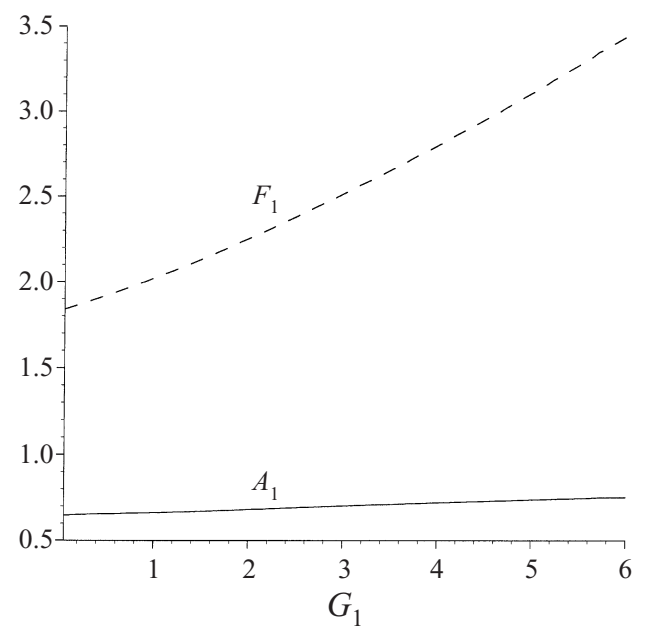

Figure 2. Dimensionless trailing film thickness, $A_{1}=C a^{-2 / 3} h_{1}$, and dimensionless pressure drop across the rear meniscus, $F_{1}=C a^{-2 / 3}\left(\Delta P_{1}-1\right)$, versus the flexibility parameter, $G_{1}$.

so that

$$
\left.\begin{array}{l}
R(0)=R_{c} \sim 1-C a^{2 / 3} \beta C_{1}+O(C a), \\
R_{z}(0)=-\tan \theta_{c} \sim-C a^{1 / 3} B_{1}+O(C a),
\end{array}\right\}
$$

i.e. to leading order $R_{c} \sim 1$ and $\theta_{c} \sim 0$. Then from (2.4) we deduce that $R_{z z}^{(0)}(0)=-1$, which in (3.20) give $\beta=A_{1}$, or equivalently,

$$
h_{1} \sim A_{1}\left(G_{1}\right) C a^{2 / 3} .
$$

Moreover, substituting for $\tan \theta_{c}$ and $R_{c}$ in (3.5) gives the first two terms in an expansion for the pressure difference across the rear meniscus:

$$
\Delta P_{1}=1+F_{1}\left(G_{1}\right) C a^{2 / 3} .
$$

Figure 2 shows plots of the dimensionless trailing film thickness, $A_{1}=h_{1} /\left(\mathrm{Ca}^{2 / 3}\right)$, and the dimensionless pressure drop across the rear bolus, $F_{1}=\left(\Delta P_{1}-1\right) C a^{-2 / 3}$, as functions of the flexibility parameter $G_{1}$. It is seen that, for fixed $C a$, both the trailing film thickness and the pressure drop across the rear meniscus increase as the wall flexibility increases. We note also that the dependence of the trailing film thickness on $G_{1}$ is approximately linear for the range of $G_{1}$ that we have considered.

This completes the analysis for the propagation of a single meniscus into a flexible tube: the propagation speed is related to the pressure difference across the meniscus by (3.23), and the thickness of the film it leaves behind is then given by (3.22). The equations of Bretherton (1961) are reproduced by (3.22) and (3.23) in the limit as $G_{1}$ tends to zero. Similarly, the lubrication analysis of Gaver et al. (1996) reduces to the present theory if wall tension is eliminated.

\subsection{The front meniscus}

Now we apply the same asymptotic arguments to the front meniscus. The same differential equation (3.15) is found for $\eta$ (here non-dimensionalized with the precursor film thickness $\left.h_{2}^{*}\right)$, with $G_{1}$ replaced by $G_{2} \equiv G_{1}\left(h_{1} / h_{2}\right)^{2}$ and the initial condition of uniform $\eta$ now imposed for large positive $\zeta$. Since (3.17) possesses two roots with negative real part, this problem admits a one-parameter family of solutions, in 
contrast with the rear meniscus. The difference in structure between the front and rear meniscus is familiar from the Landau-Levich equation (Bretherton 1961) but here the qualitative behaviour of the free surface depends on the size of $G_{2}$. For $G_{2}<\left(\frac{3}{4}\right)^{1 / 3}$, the solution for $\eta$ oscillates and decays as $\zeta \rightarrow \infty$, as for the Landau-Levich equation:

$$
\eta \sim 1+\alpha \mathrm{e}^{-\zeta / 2 \tilde{l}^{2}} \cos \left(\frac{\zeta \sqrt{4 \tilde{l}^{3}-1}}{2 \tilde{l}^{2}}\right) \text { as } \zeta \rightarrow \infty, \quad \tilde{l}>4^{-1 / 3} .
$$

( $\tilde{l}$ is the real positive solution of (3.17) with $G_{1}$ replaced by $G_{2}$.) When $G_{2}>\left(\frac{3}{4}\right)^{1 / 3}$ the free-surface profile decays monotonically at infinity:

$$
\eta \sim 1+\alpha \mathrm{e}^{-\zeta / 2 \tilde{l}^{2}} \cosh \left(\frac{\zeta \sqrt{1-4 \tilde{l}^{3}}}{2 \tilde{l}^{2}}\right) \quad \text { as } \quad \zeta \rightarrow \infty, \quad \tilde{l}<4^{-1 / 3} .
$$

In (3.24) and (3.25) $\alpha$ is an arbitrary constant which parameterizes the solution space. Once again we expect the transition film to behave quadratically as it approaches the meniscus:

$$
\eta \sim \frac{1}{2} A_{2} \zeta^{2}+B_{2} \zeta+C_{2} \quad \text { as } \quad \zeta \rightarrow-\infty .
$$

Matching this parabola with the front meniscus as before gives

$$
h_{2} \sim A_{2} \mathrm{Ca}^{2 / 3},
$$

and by analogy with (3.23) the pressure difference across the front meniscus is given by

$$
\Delta P_{2} \sim 1+F_{2} C a^{2 / 3}
$$

where $F_{2}=A_{2} C_{2}-\left(\frac{1}{2}\right) B_{2}^{2}$.

Recall that $h_{2}^{*}$, unlike $h_{1}^{*}$, is a physically specified quantity. Once $h_{2}$ and $C a$ are specified, (3.27) is an equation for $A_{2}$. One can envisage the following solution procedure, which is that traditionally applied to the Landau-Levich equation. For each value of $G_{2}$, solve (3.15) as an initial-value problem using either (3.24) or (3.25) as the initial condition. By varying the free parameter $\alpha$ generate a family of such solutions. For each, read off the behaviour as $\zeta \rightarrow-\infty$ and thus find $A_{2}$ and $F_{2}$. Hence plot $F_{2}$ versus $A_{2}$ for each fixed $G_{2}$, using $\alpha$ to parameterize the curve.

Unfortunately, while this procedure is straightforward for the Landau-Levich problem (i.e. with $G_{2}=0$ ), it becomes increasingly problematic as $G_{2}$ is increased towards its critical value of $\left(\frac{3}{4}\right)^{1 / 3}$. An increasingly large portion of the solution space corresponds to solutions in which the film thickness reaches zero at a finite value of $\zeta$, so that the behaviour (3.28) is never realized. For $G_{2}>\left(\frac{3}{4}\right)^{1 / 3}$ we have failed to obtain any solutions of the initial value problem (3.15), (3.25) in which $h$ is uniformly non-zero by this method.

Therefore, we are forced to shoot the other way, starting at large negative $\zeta$. For each value of $A_{2}$ we vary $F_{2}$ as a shooting parameter until the desired constant behaviour of $\eta$ as $\zeta \rightarrow \infty$ is obtained. This procedure for constructing $F_{2}$ as a function of $A_{2}$ is rather more laborious than that described above, but does not suffer from the same difficulties as $G_{2}$ is increased.

In figure $3(a)$ we plot $F_{2}$ versus $A_{2}$ for several different values of $G_{2}$. The graph with $G_{2}=0$ is the classical Landau-Levich curve which can be found for example in Wong et al. (1995a). It shows that the pressure drop across the front meniscus is an increasing function of the precursor thickness. As the flexibility is increased, we see that the pressure jump is increased. 

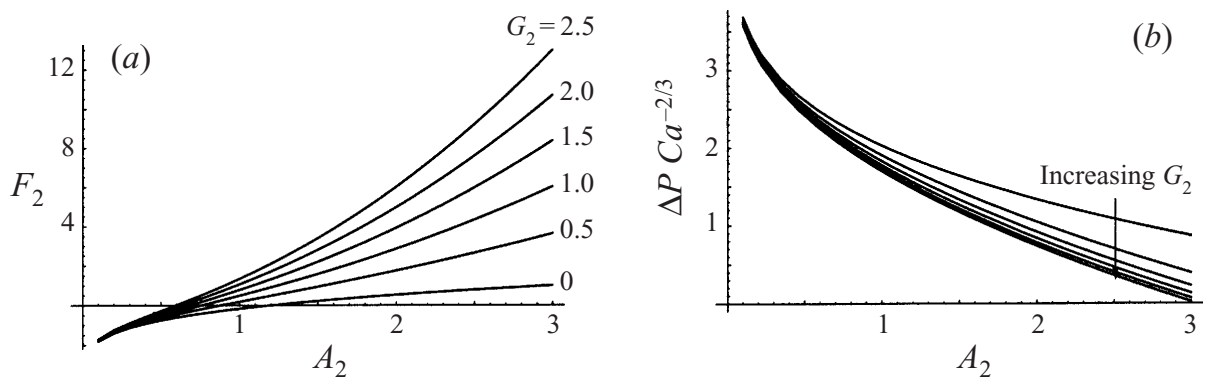

FIGURE 3. Dimensionless pressure drop versus dimensionless precursor thickness, $A_{2}=\mathrm{Ca}^{-2 / 3} h_{2}$, for various values of the flexibility parameter, $G_{2}=0,0.5,1.0,1.5,2.0,2.5:(a)$ across the front meniscus, $F_{2}=C a^{-2 / 3}\left(\Delta P_{2}-1\right) ;(b)$ across the bolus, $F_{1}-F_{2}=C a^{-2 / 3} \Delta P$.

\subsection{Governing equations for the bolus}

From (3.23) and (3.28), the total pressure drop driving the bolus is related to its propagation speed by

$$
\Delta P \sim\left[F_{1}\left(G_{1}\right)-F_{2}\left(G_{2} ; A_{2}\right)\right] C a^{2 / 3} .
$$

For a rigid tube, when $G_{1} \equiv G_{2} \equiv 0$, this means that, given the precursor layer thickness $h_{2}$ and the imposed pressure drop $\Delta P$, the propagation speed of the bolus is given by (3.29). Then the trailing film thickness $h_{1}$ can be found from (3.22). In general the situation is complicated by the fact that (3.29) and (3.22) are coupled through the appearance of $h_{1}$ in the definition of $G_{1}$. However, notice that $G_{1}$ and $G_{2}$ are related via

$$
A_{1}^{2}\left(G_{1}\right) G_{1} \equiv A_{2}^{2} G_{2} .
$$

Given $A_{2}$ and $G_{2}, G_{1}$ is found by solving the implicit equation (3.30) numerically. Then $F_{1}\left(G_{1}\right)$, and hence from (3.29) the total pressure drop across the bolus, can be found as a function of $A_{2}$ and $G_{2}$. The dimensionless pressure drop is plotted against $A_{2}$ in figure $3(b)$ for several values of $G_{2}$. The figure shows that for fixed capillary number and precursor thickness, the pressure difference decreases as the wall flexibility increases. In other words, increasing wall flexibility makes the bolus easier to force along the tube. For fixed values of $G_{2}$ and capillary number the pressure difference decreases as the precursor film thickness, $A_{2}$, increases. Hence the thicker the precursor film the easier it is to force the bolus along the tube.

This result could not have been predicted trivially. The pressure drop across either of the two menisci is increased with increasing wall flexibility. We have found that the drop across the front meniscus is increased more. The effect is slight compared to the effect of precursor thickness; however, recall that the current analysis is only valid for slightly flexible tubes, $\Gamma \sim O\left(\mathrm{Ca}^{2 / 3}\right)$.

\subsection{Criterion for eventual rupture}

The rate at which the volume of the bolus, $V^{*}$, changes is given to leading order by

$$
\frac{\mathrm{d} V^{*}}{\mathrm{~d} t^{*}} \sim 2 \pi a U\left(h_{2}^{*}-h_{1}^{*}\right)
$$

which non-dimensionalized gives the dimensionless flow rate, $Q$, as

$$
Q=\frac{3 \mu}{2 \pi a^{2} \sigma} \frac{\mathrm{d} V}{\mathrm{~d} t} \sim C a\left(h_{2}-A_{1} C a^{2 / 3}\right) .
$$




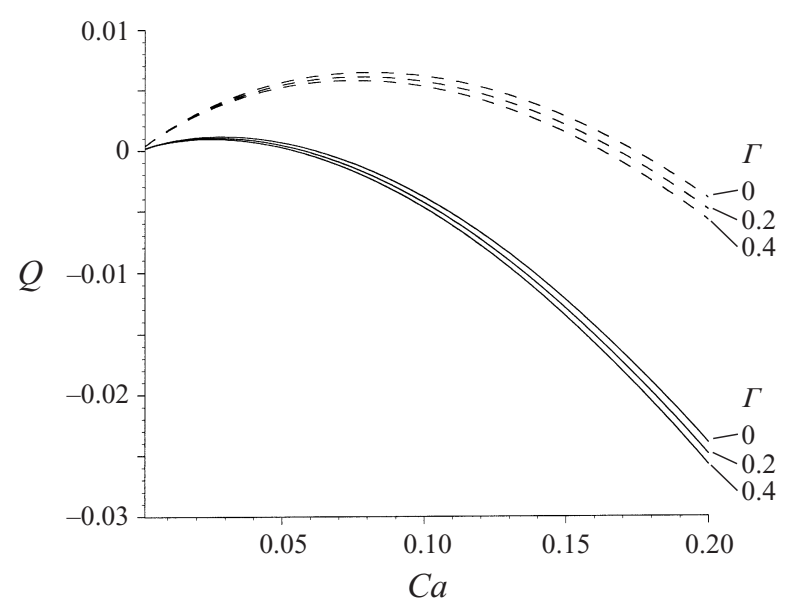

Figure 4. Dimensionless rate of change of bolus volume, $Q=\left(3 \mu / 2 \pi a^{2} \sigma\right) \mathrm{d} V / \mathrm{d} t$, versus capillary number for various values of the compliance parameter, $\Gamma$. Solid lines: $h_{2}=0.1$; dashed lines: $h_{2}=0.2$.

(a)

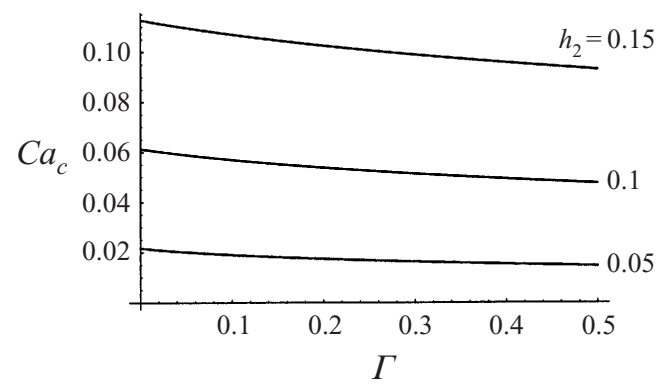

(b)

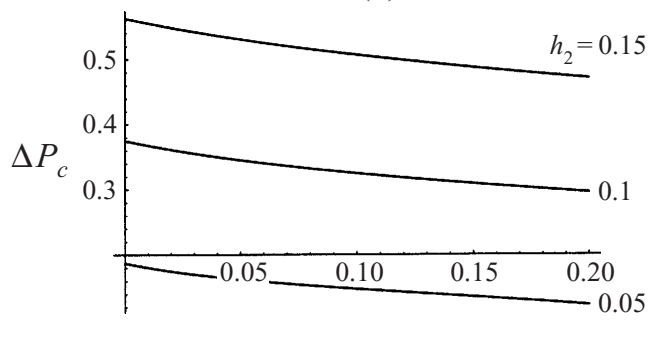

$\Gamma$

FIGURE 5. (a) Critical capillary number and (b) critical dimensionless pressure drop versus compliance parameter, $\Gamma$, for various values of the dimensionless precursor thickness, $h_{2}$.

The dimensionless rate of change of bolus volume is plotted against capillary number for different values of the precursor thickness, $h_{2}$, and compliance, $\Gamma$, in figure 4 . In each case, there is a critical capillary number above which $h_{1}>h_{2}$, so that the bolus volume decreases as the bolus propagates. This gives a minimum criterion for eventual tube reopening. The critical capillary number can be written in the form

$$
C a_{c}=h_{2}^{3 / 2} f\left(\frac{1}{h_{2}} \Gamma\right)
$$

where $f$ satisfies

$$
f(\xi)^{2 / 3} A_{1}\left(\xi f(\xi)^{2 / 3}\right) \equiv 1 .
$$

The critical capillary number is plotted against $\Gamma$ for various precursor thicknesses in figure $5(a)$; it is seen to decrease with increasing wall flexibility. The effect is very slight compared to the effect of precursor thickness.

From (3.29), we can deduce a critical pressure drop which must be exceeded for $\mathrm{Ca}$ to exceed $\mathrm{Ca}$ so that the airway will ultimately reopen. The critical pressure drop 

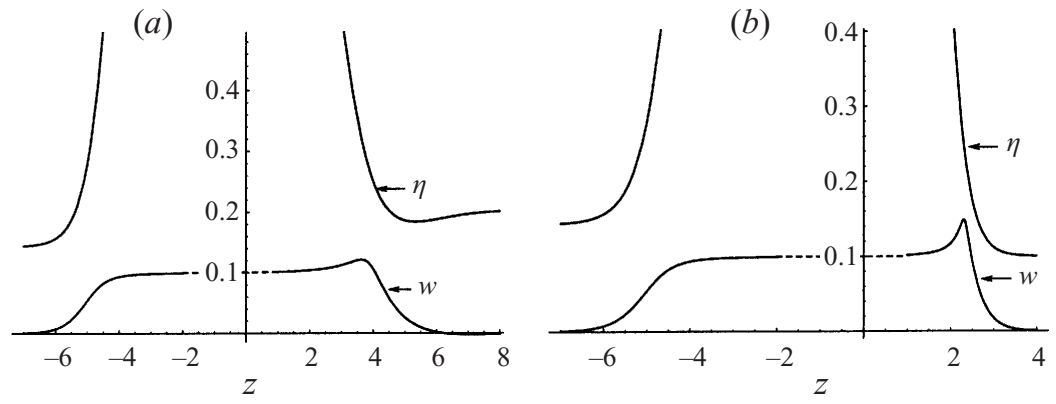

FIgURE 6. Solution for the film height and wall displacement ahead of and behind the bolus. (a) $\Gamma=0.1, C a=0.1, h_{2}=0.2 ;(b) \Gamma=0.1, C a=0.1, h_{2}=0.1$.

takes the form

where $g$ is given by

$$
\Delta P_{c}=h_{2} g\left(\frac{1}{h_{2}} \Gamma\right)
$$

$$
g(\xi) \equiv f(\xi)^{2 / 3}\left[F_{1}\left(\xi f(\xi)^{2 / 3}\right)-F_{2}\left(\xi f(\xi)^{2 / 3} ; A_{1}\left(\xi f(\xi)^{2 / 3}\right)\right)\right] .
$$

The critical reopening pressure drop is plotted against $\Gamma$ for various precursor thicknesses in figure $5(b)$. There is a decrease in the reopening pressure drop as the wall flexibility increases. In other words a more flexible airway is easier to reopen. For a fixed value of $\Gamma$ the critical reopening pressure drop increases as the precursor film thickness increases. This is an interesting contrast to the dependence of the pressure drop across the bolus on the precursor film thickness (figure $3 b$ ). We also consider the capillary number as a function of the pressure drop across the bolus. As expected, the speed of the bolus increases as the pressure drop across it increases. Moreover, for a given pressure drop, we find that the capillary number, and hence the bolus propagation speed, is slightly greater in a more compliant tube (for example, when $\Gamma=0$ we find that $C a \approx 0.03+0.24 \Delta P$ and when $\Gamma=0.4$ we have that $C a \approx 0.04+0.24 \Delta P$ ).

Finally, in figure 6 we plot the air-liquid and liquid-wall interfaces for different values of the wall compliance and the capillary number. In both cases the compliance parameter and capillary number are the same: $\Gamma=0.1, C a=0.1$. In figure $6(a)$, we have a relatively thick precursor with $h_{2}=0.2$. The corresponding value of $G_{2}$ is $G_{2} \approx 0.5386<\left(\frac{3}{4}\right)^{1 / 3}$, and so we observe that the wall and free surface exhibit oscillations ahead of the bolus. The trailing film thickness here is $h_{1} \approx 0.1398<h_{2}$, so this bolus will grow as it propagates. In figure $6(b)$ we decrease the precursor thickness to $h_{2}=0.1$. Since the values of $\Gamma$ and $C a$ are unchanged, the rear mensicus is identical to that in figure 6(a). In particular we still have that $h_{1} \approx 0.1398>h_{2}$ so that this bolus will shrink as it propagates. Here $G_{2} \approx 2.154>\left(\frac{3}{4}\right)^{1 / 3}$ so the film profile $\eta$ decays monotonically ahead of the bolus. However we observe that the wall displacement still has a maximum. Note that, in both cases, there is a region of relatively rapid change in the wall displacement ahead of the bolus. In the following section we shall show that longitudinal wall tension smooths out this region.

\section{Tension dominated case}

In $\S 3$ we considered the longitudinal wall tension, $T$, to be much less than 1 . Here we consider the limiting case in which the longitudinal wall tension is much greater 
than the wall compliance. Unlike $\S 3$, we do not assume that the wall compliance is of order $C a^{2 / 3}$, but only that it is small.

Since we retain the longitudinal curvature term, of order $\hat{T}=\Gamma T$, in the wall law (2.8) but neglect nonlinear terms of $O\left(\Gamma^{2}\right)$ we must have that $\hat{T}=\Gamma T \gg \Gamma^{2}$. We treat $\hat{T}$ as a small parameter, such that $C a^{2 / 3} \ll \hat{T} \ll 1$, and hence our analysis is for $\Gamma \ll T \ll \Gamma^{-1}$. The solution is expressed as a double asymptotic expansion and, in this limit, the leading-order solutions for the trailing film thickness and the pressure drop across the bolus depend only on $C a$ and $T$.

\subsection{Quasi-steady problem for a meniscus}

We employ the dimensionless linear wall law, (2.8), relating the wall displacement, $w(z)$, to the pressure, $P$, repeated here for convenience:

$$
-w+\hat{T} w_{z z}=1+P,
$$

where $\hat{T}=\Gamma T$. The internal pressure, $P$, and wall displacement, $w$, are scaled as in $\S 2$. Since we have assumed that the meniscus meets the wall at $z=0$, immediately to the right of $z=0$ the wall therefore sees a uniform internal pressure $P_{b}$, while immediately to the left the effective internal pressure is $a P_{1} / \sigma-\kappa$, where $\kappa$ is the wall curvature. The second term of the effective internal pressure is due to the liquid film left behind by the meniscus, which to leading order in $\mathrm{Ca}$ has zero thickness but nevertheless a finite surface tension. The film thickness, $h$, is scaled with the trailing film thickness $h_{1}$ and we anticipate the scaling

$$
h_{1}=\beta_{1} C a^{2 / 3},
$$

where $\beta_{1}$ is an order-one constant to be determined. The problem for the wall displacement $w$ is thus

$$
\begin{aligned}
-(1-\Gamma) w+(\hat{T}+\Gamma) w_{z z}=-\beta_{1} C a^{2 / 3} h-\beta_{1} C a^{2 / 3} h_{z z} & \\
+\beta_{1} C a^{2 / 3} h \Gamma w_{z}\left(\Gamma w_{z}+\beta_{1} C a^{2 / 3} h_{z}+\Gamma w_{z z z}+\beta_{1} C a^{2 / 3} h_{z z z}\right), & z<0, \\
-w+\hat{T} w_{z z}=1-\Delta P_{1}, & z>0,
\end{aligned}
$$

where, as before, $\Delta P_{1}=P_{1}-P_{b}$. The boundary conditions for (4.3) are that $w$ be finite as $|z| \rightarrow \infty$ and that $w$ and $w_{z}$ be continuous at $z=0$.

\subsection{Outer region}

Since $C a$ is the smallest parameter of the problem, we consider first a regular perturbation expansion for $w$ in powers of $C a^{1 / 3}$. The terms of this expansion are then themselves expanded regularly in powers of $\hat{T}$ and $\Gamma$. Substitution of these expansions into $(4.3 a, b)$ gives that to leading order $w=$ const. in $z<0$, and likewise in $z>0$ (i.e. in the bolus), with these constants being

$$
\begin{array}{ll}
w=\beta_{1} C a^{2 / 3}+O\left(\Gamma C a^{2 / 3}, C a\right), & z<0, \\
w=w_{b}\left(\Delta P_{1}\right)=\Delta P_{1}-1, & z>0 .
\end{array}
$$

These outer solutions do not satisfy the conditions that both $w$ and $w_{z}$ be continuous at $z=0$. Instead we must investigate two transition regions: an intermediate region, of length $a \sqrt{\hat{T}}$ (found by performing a leading-order balance in (4.3)), which is the elastic transition region over which the wall deflection adjusts to the different pressures exerted on it; and an inner region, of length $a C a^{1 / 3}$ (as in $\S 3$ ), which is the fluid transition region over which the fluid surface curvature adjusts. In this 
tension dominated limit, the length scales of the two regions are partially decoupled. Moreover, the length scales of the regions force the expansions for the dependent variables in the regions to be in powers of $C a^{1 / 3} / \sqrt{\hat{T}}$.

\subsection{Intermediate region}

There is a boundary layer near $z=0$ in which the two constants, given in (4.4), are smoothly joined. To examine this region, we rescale as follows:

$$
z=\sqrt{\hat{T}} \xi, \quad w(z) \equiv \tilde{w}(\xi), \quad h(z) \equiv \tilde{h}(\xi),
$$

so that (4.3) becomes

$$
\begin{aligned}
-(1-\Gamma) \tilde{w}+(1+\bar{\alpha}) \tilde{w}_{\xi \xi}=-\beta_{1} C a^{2 / 3} \tilde{h}-\frac{\beta_{1} C a^{2 / 3}}{\hat{T}} \tilde{h}_{\xi \xi} & \\
+\frac{\beta_{1} C a^{2 / 3}}{\hat{T}} \tilde{h} \Gamma \tilde{w}_{\xi}\left(\Gamma \tilde{w}_{\xi}+\beta_{1} C a^{2 / 3} \tilde{h}_{\xi}+\bar{\alpha} \tilde{w}_{\xi \xi \xi}+\frac{\beta_{1} C a^{2 / 3}}{\hat{T}} \tilde{h}_{\xi \xi \xi}\right), & \xi<0, \\
-\tilde{w}+\tilde{w}_{\xi \xi}=1-\Delta P_{1}, & \xi>0,
\end{aligned}
$$

where $\bar{\alpha}=\Gamma / \hat{T}=1 / T$. We can derive an equation relating the film thickness and wall displacement:

$$
(1-\Gamma \tilde{w})\left(\Gamma \tilde{w}_{\xi}+\beta_{1} C a^{2 / 3} \tilde{h}_{\xi}+\bar{\alpha} \tilde{w}_{\xi \xi \xi}+\frac{\beta_{1} C a^{2 / 3}}{\hat{T}} \tilde{h}_{\xi \xi \xi}\right) \tilde{h}^{3}-\frac{\sqrt{\hat{T}}}{\beta_{1}^{2} C a^{1 / 3}} \tilde{h}(1-\Gamma \tilde{w})=\text { const. }
$$

We assume an expansion for the wall displacement, film thickness and pressure drop, in this intermediate region, of the general form

$$
\begin{aligned}
\left(\tilde{w}(\xi), \tilde{h}(\xi), \Delta P_{1}\right) \sim\left(\tilde{w}^{(0)}, \tilde{h}^{(0)}, \Delta P_{1}^{(0)}\right) & +\frac{\alpha \beta_{1} C a^{1 / 3}}{\sqrt{\hat{T}}}\left(\tilde{w}^{(1)}, \tilde{h}^{(1)}, \Delta P_{1}^{(1)}\right) \\
& +\frac{\alpha^{2} \beta_{1}^{2} C a^{2 / 3}}{\hat{T}}\left(\tilde{w}^{(2)}, \tilde{h}^{(2)}, \Delta P_{1}^{(2)}\right)+\cdots,
\end{aligned}
$$

where $\alpha$ is a second order-one constant to be determined later.

The leading-order equations for the wall displacement are thus

$$
\begin{array}{rr}
-(1-\Gamma) \tilde{w}^{(0)}+(1+\bar{\alpha}) \tilde{w}_{\xi \xi}^{(0)}=0, & \xi<0, \\
-\tilde{w}+\tilde{w}_{\xi \xi}^{(0)}=1-\Delta P_{1}^{(0)}, & \xi>0,
\end{array}
$$

and for the film thickness

$$
\left(1-\Gamma \tilde{w}^{(0)}\right) \tilde{h}^{(0)}=\text { const. }
$$

The leading-order boundary conditions are

$$
\begin{aligned}
& \left|\tilde{w}^{(0)}\right|<\infty \text { as }|\xi| \rightarrow \infty, \\
& \tilde{w}^{(0)}, \tilde{w}_{\xi}^{(0)} \text { continuous at } \xi=0, \\
& h^{(0)} \rightarrow 1 \text { as } \xi \rightarrow-\infty .
\end{aligned}
$$

Equations (4.9) together with the boundary conditions given by $(4.11 a, b)$ may be 


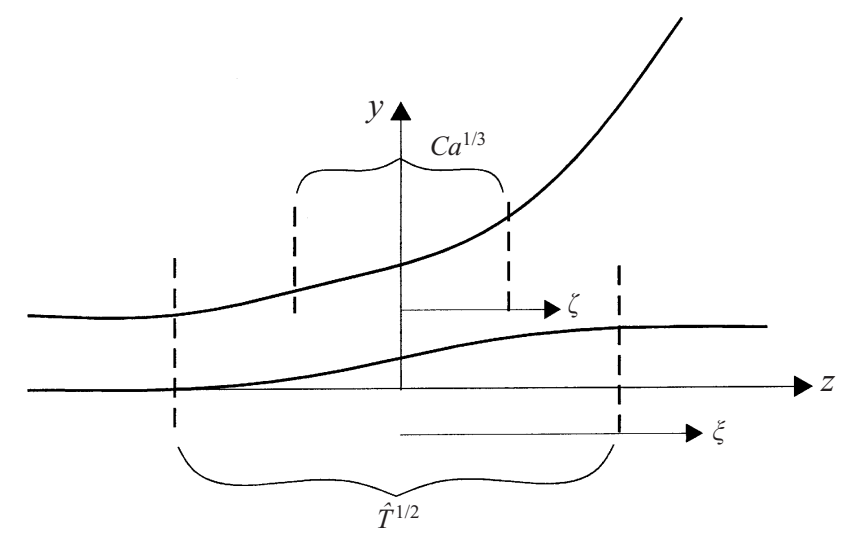

FIGURE 7. Definition sketch of the intermediate- and inner-region length scales in the tension dominated case.

solved to give

$$
\begin{array}{ll}
\tilde{w}^{(0)} \sim\left(\Delta P_{1}^{(0)}-1\right) \frac{\sqrt{1+\bar{\alpha}}}{1+\sqrt{1+\bar{\alpha}}} \exp \left(\frac{\xi}{\sqrt{1+\bar{\alpha}}}\right)+O(\Gamma), \quad \xi<0, \\
\tilde{w}^{(0)} \sim\left(\Delta P_{1}^{(0)}-1\right)\left(1-\frac{1}{1+\sqrt{1+\bar{\alpha}}} \mathrm{e}^{-\xi}\right)+O(\Gamma), \quad \xi>0 .
\end{array}
$$

In terms of this solution, we define the constants:

$$
\begin{aligned}
& W_{0}:=\tilde{w}^{(0)}(0) \sim\left(\Delta P_{1}^{(0)}-1\right) \frac{\sqrt{1+\bar{\alpha}}}{1+\sqrt{1+\bar{\alpha}}}+O(\Gamma), \\
& W_{1}:=\tilde{w}_{\xi}^{(0)}(0) \sim\left(\Delta P_{1}^{(0)}-1\right) \frac{1}{1+\sqrt{1+\bar{\alpha}}}+O(\Gamma),
\end{aligned}
$$

as functions of $\Delta P_{1}^{(0)}$.

Since $\tilde{h}^{(0)} \rightarrow 1, \tilde{w}^{(0)} \rightarrow 0$ as $\xi \rightarrow-\infty$, from (4.10) the leading-order trailing film thickness is

$$
\tilde{h}^{(0)}(\xi)=\frac{1}{1-\Gamma \tilde{w}^{(0)}(\xi)} .
$$

Thus, unlike $\S 3$, because of the non-trivial deflection of the tube walls, the leadingorder film thickness is non-uniform in this region and simply adjusts so as to conserve mass.

The spherical cap cannot be joined analytically to the thin film behind the bolus (4.14) and so, as in $\S 3.2$, we now consider the transition region between the meniscus and the trailing film. This is referred to as the inner region and in this region viscous forces are important. The relationship between the inner and intermediate regions is shown in figure 7 where we see that the wall deformation occurs over a larger length scale than the fluid-surface deformation.

\subsection{The inner region}

In this region, following (3.6) we rescale $z$ via

$$
z=\alpha h_{1} C a^{-1 / 3} \zeta
$$


We also rescale $w$ via $w \equiv W(\zeta)$. From (4.14) the film thickness to which the inner region must match as $\zeta \rightarrow-\infty$ is $h_{1}^{*} /\left(1-\Gamma W_{0}\right)$, so this is used to non-dimensionalize the film thickness in this inner region: $h \equiv H(\zeta) /\left(1-\Gamma W_{0}\right)$.

The governing film and wall equations are

$$
\begin{gathered}
(1-\Gamma W) K_{\zeta} H^{3}-\frac{\alpha}{\beta_{1}}\left(1-\Gamma W_{0}\right)^{2}(1-\Gamma W) H=\text { const. } \\
-W+\frac{\hat{T}}{\alpha^{2} \beta_{1}^{2} C a^{2 / 3}} W_{\zeta \zeta}=1-\mathrm{K}+\Gamma \delta H W_{\zeta} \mathrm{K}_{\zeta},
\end{gathered}
$$

respectively, where for simplicity we introduce the shorthand $\delta=\left(\alpha^{2} \beta_{1}\left(1-\Gamma W_{0}\right)\right)^{-1}$.

Our expansions for the wall displacement and the film thickness in this inner region take the general form

$$
\begin{aligned}
\left(w,\left(1-\Gamma W_{0}\right) h\right)= & (W(\zeta), H(\zeta)) \sim\left(W^{(0)}, H^{(0)}\right)+\frac{\alpha \beta_{1} C a^{1 / 3}}{\sqrt{\hat{T}}}\left(W^{(1)}, H^{(1)}\right) \\
& +\frac{\alpha^{2} \beta_{1}^{2} C a^{2 / 3}}{\hat{T}}\left(W^{(2)}, H^{(2)}\right)+\frac{\alpha^{3} \beta_{1}^{3} C a}{\hat{T}^{3 / 2}}\left(W^{(3)}, H^{(3)}\right)+\cdots .
\end{aligned}
$$

Substituting (4.17) into (4.16), we obtain the leading-order equations

$$
W_{\zeta \zeta}^{(0)}=0, \quad W_{\zeta \zeta}^{(1)}=0 .
$$

Hence, $W^{(0)}$ and $W^{(1)}$ are linear in $\zeta$. In order to determine their exact form we must match this inner solution to the intermediate solution found in the previous section.

\subsection{Matching}

We now match the expression for $w$ in the inner region (4.17) with the expression in the intermediate region (4.8). We find that $W^{(0)}=W_{0}$ and $W^{(1)}=\lambda+W_{1} \zeta$ where $W_{0}$ and $W_{1}$ are defined by (4.13) and $\lambda$ is an as yet unknown constant.

Finally, we have that the free-surface displacement in the inner region, $1-\Gamma W-$ $\beta_{1} \mathrm{Ca}^{2 / 3} \mathrm{H}$, must match, as $\zeta \rightarrow \infty$, to the outer, capillary static solution. We expand the equation of the spherical cap, $R$, about $z=0$ as in $\S 3.3: \beta$ in (3.19) is replaced here by $\alpha \beta_{1}$. In order to determine the leading-order (in $C a$ ) effective contact radius and contact angle we do not need to determine the leading-order expression for the film thickness in the inner region, $H^{(0)}$, at this stage. Matching the outer, capillary static solution with the expressions for $W^{(0)}$ and $W^{(1)}, R_{c}$ and $\tan \theta_{c}$ are given asymptotically by

$$
\left.\begin{array}{l}
R_{c}=R^{(0)}(0)+\cdots=1-\Gamma W_{0}+\cdots, \\
\tan \theta_{c}=-R_{z}^{(0)}(0)+\cdots=\sqrt{\bar{\alpha} \Gamma} W_{1}+\cdots .
\end{array}\right\}
$$

Using (3.5), the leading-order (in $C a$ ) pressure jump across the meniscus is

$$
\Delta P_{1}^{(0)}=\frac{2}{\left(1-\Gamma W_{0}\right) \sqrt{1+\bar{\alpha} \Gamma W_{1}^{2}}},
$$

which is valid up to $O\left(\Gamma^{2}\right)$. Substituting from (4.13) the leading-order (in $\Gamma$ ) expressions for $W_{0}$ and $W_{1}$ gives

$$
\Delta P_{1}^{(0)} \sim 2+\Gamma+O\left(\Gamma^{2}\right) .
$$

We shall show (§4.8) that the expression for the leading-order pressure drop across the front meniscus is identical to (4.21). Thus in order to determine the total pressure 
drop across the bolus we must determine the higher-order pressure corrections. This we do in the following subsections.

\subsection{First-order correction}

\section{Intermediate region}

First we obtain higher-order solutions to the governing equations in the intermediate region (equations (4.6) and (4.7)). To determine the first perturbation to the pressure drop it is unnecessary to compute the first perturbation to the film thickness, $\tilde{h}^{(1)}$, at this stage. The equations for $\tilde{w}^{(1)}$ are obtained by plugging the expansion (4.8) into (4.6) and considering terms of $O\left(\mathrm{Ca}^{1 / 3}\right)$. The result is two second-order o.d.e.s for $\tilde{w}^{(1)}(\xi)$ : one in $\xi<0$, and one in $\xi>0$ that also involves the unknown $\Delta P_{1}^{(1)}$. In either case, one constant of integration is set by the condition that $\tilde{w}^{(1)}(\xi)$ be finite as $\xi \rightarrow \pm \infty$, and the other is determined once $\tilde{w}^{(1)}(0 \pm)$ is specified. Hence, given $\Delta P_{1}^{(1)}$, we can deduce a relation between $\tilde{w}_{\xi}^{(1)}(0-)$ and $\tilde{w}^{(1)}(0-)$, and a second relation between $\tilde{w}_{\xi}^{(1)}(0+)$ and $\tilde{w}^{(1)}(0+)$. These relationships are

$$
\begin{aligned}
& (1-\Gamma) W_{0} \tilde{w}^{(1)}(0-) \frac{1}{1+\bar{\alpha}}=W_{1} \tilde{w}_{\xi}^{(1)}(0-), \\
& \tilde{w}^{(1)}(0+)\left[W_{0}+1-\Delta P_{1}^{(0)}\right]=W_{1} \tilde{w}_{\xi}^{(1)}(0+)+\Delta P_{1}^{(1)} \Phi_{1},
\end{aligned}
$$

where

$$
\Phi_{1}=\left(1-\Delta P_{1}^{(0)}\right) \frac{\mathrm{d} w_{b}}{\mathrm{~d} \Delta P_{1}}+\left(W_{0}-w_{b}\right)+w_{b} \frac{\mathrm{d} w_{b}}{\mathrm{~d} \Delta P_{1}}\left(\Delta P_{1}^{(0)}\right) .
$$

As before, we now investigate the inner fluid transition region.

\section{Inner region}

Proceeding to the next order in the governing equations (4.16), at $O(1)$ we obtain the equations

$$
\begin{aligned}
& \left(1-\Gamma W_{0}\right)\left(\bar{\alpha} W_{\zeta \zeta \zeta}^{(2)}+\delta H_{\zeta \zeta \zeta}^{(0)}\right) H^{(0)^{3}}-\frac{\alpha}{\beta_{1}}\left(1-\Gamma W_{0}\right)^{3} H^{(0)}=\text { const., } \\
& -(1-\Gamma) W^{(0)}+(1+\bar{\alpha}) W_{\zeta \zeta}^{(2)}=-\delta H_{\zeta \zeta}^{(0)} .
\end{aligned}
$$

From (4.24b), using the fact that $W_{0}$ is constant, we get

$$
W_{\zeta \zeta \zeta}^{(2)}=-\frac{\delta}{(1+\bar{\alpha})} H_{\zeta \zeta \zeta}^{(0)}
$$

Combining (4.25) with (4.24a), we get a single third-order o.d.e. for $H^{(0)}$. By choosing

$$
\alpha=\left(\frac{1}{(1+\bar{\alpha})^{1 / 3}\left(1-\Gamma W_{0}\right)}\right),
$$

this is reduced to the Landau-Levich equation, i.e.

$$
H^{(0)^{3}} H_{\zeta \zeta \zeta}^{(0)}=H^{(0)}-1, \quad H^{(0)} \rightarrow 1 \quad \text { as } \zeta \rightarrow-\infty .
$$

This problem is solved numerically as described in $\S 3$, and yields

$$
H^{(0)} \sim \frac{1}{2} A_{1} \zeta^{2}+B_{1} \zeta+C_{1} \text { as } \zeta \rightarrow \infty,
$$


where $A_{1}, B_{1}$ and $C_{1}$ are numerically determined constants: exactly the same constants as those for the classical stiff tube analysis of Bretherton (1961), equivalent to setting $G_{1}=0$ in $\S 3$. From equation $(4.24 b)$, we see that the asymptotic behaviour of the inner solution for the wall displacement is of the form

$$
\begin{aligned}
& W^{(2)} \sim \frac{1}{2} \tilde{a}_{1} \zeta^{2}+\tilde{b}_{1} \zeta+\tilde{c}_{1} \quad \text { as } \quad \zeta \rightarrow-\infty \\
& W^{(2)} \sim \frac{1}{2} a_{1} \zeta^{2}+b_{1} \zeta+c_{1} \quad \text { as } \quad \zeta \rightarrow+\infty
\end{aligned}
$$

\section{Matching}

As in $\S 4.5$ we match the intermediate and inner expansions for the wall displacement, $\tilde{w}$ and $W$ respectively, and hence we deduce the following relationships between the constants:

$$
\left.\begin{array}{llll}
\lambda=\tilde{w}^{(1)}(0-), & \tilde{a}_{1}=\tilde{w}_{\xi \xi}^{(0)}(0-), & \tilde{b}_{1}=\tilde{w}_{\xi}^{(1)}(0-), & \tilde{c}_{1}=\tilde{w}^{(2)}(0-), \\
\lambda=\tilde{w}^{(1)}(0+), & a_{1}=\tilde{w}_{\xi \xi}^{(0)}(0+), & b_{1}=\tilde{w}_{\xi}^{(1)}(0+), & c_{1}=\tilde{w}^{(2)}(0+) .
\end{array}\right\}
$$

Furthermore, by integrating (4.24b) twice with respect to $\zeta$ and using the expressions for $W^{(2)}$ and $H^{(0)}$ at $\pm \infty$ (equations (4.27), (4.28) and (4.29)) we also get

$$
\begin{aligned}
& A_{1}=\frac{1}{\delta}\left(-(1+\bar{\alpha}) \tilde{w}_{\xi \xi}^{(0)}(0+)+W_{0}(1-\Gamma)\right), \\
& B_{1}=(1+\bar{\alpha})\left(\tilde{b}_{1}-b_{1}\right) \alpha^{2} \beta_{1}\left(1-\Gamma W_{0}\right), \\
& C_{1}=1+(1+\bar{\alpha})\left(\tilde{c}_{1}-c_{1}\right) \alpha^{2} \beta_{1}\left(1-\Gamma W_{0}\right) .
\end{aligned}
$$

From $(4.9 b)$

$$
\tilde{w}_{\xi \xi}^{(0)}(0+)=1-\Delta P_{1}^{(0)}+\tilde{w}^{(0)}(0+) .
$$

Thus, substituting the above into $(4.31 a)$ we obtain the following expression for $\beta_{1}$ and hence for the trailing film thickness:

$$
\frac{h_{1}}{C a^{2 / 3}}=\beta_{1} \sim \frac{A_{1}}{\alpha^{2}\left(1-\Gamma W_{0}\right)\left[-(1+\bar{\alpha})\left(1-\Delta P_{1}^{(0)}+W_{0}\right)+W_{0}(1-\Gamma)\right]} .
$$

The final step is to match the inner solution as $\zeta \rightarrow \infty$ to the outer, capillary static solution. The effective contact radius and contact angle for the meniscus are then given asymptotically by

$$
\begin{aligned}
R_{c} & \sim 1-\Gamma W_{0}-\alpha \beta_{1} C a^{1 / 3} \sqrt{\bar{\alpha} \Gamma} \lambda-\alpha^{2} \beta_{1}^{2} C a^{2 / 3}\left(\bar{\alpha} c_{1}+\delta C_{1}\right)+\cdots, \\
\tan \theta_{c} & \sim \sqrt{\bar{\alpha} \Gamma} W_{1}+\alpha \beta_{1} C a^{1 / 3}\left(\bar{\alpha} b_{1}+\delta B_{1}\right)+\cdots,
\end{aligned}
$$

and hence, from (3.5), the first perturbation to the pressure drop across the meniscus is

$$
\Delta P_{1}^{(1)}=\frac{2 \Gamma}{\left(1-\Gamma W_{0}\right)^{2} \sqrt{1+\bar{\alpha} \Gamma W_{1}^{2}}}\left(\lambda-\frac{W_{1} B_{1}+\alpha^{2} \beta_{1} W_{1} b_{1} \bar{\alpha}\left(1-\Gamma W_{0}\right)}{\alpha^{2} \beta_{1}\left(1+\bar{\alpha} \Gamma W_{1}^{2}\right)}\right) .
$$

From (4.22) and (4.31b) (using (4.30)) we thus determine $b_{1}, \tilde{b_{1}}, \lambda$ in terms of $B_{1}$ and $\Delta P_{1}^{(1)}$. When these expressions are substituted into equation (4.35) for the pressure drop, $\Delta P_{1}^{(1)}$, we get

$$
\left(1-2 \Gamma+O\left(\Gamma^{2}\right)\right) \Delta P_{1}^{(1)}=0,
$$

and since the term in brackets is non-zero we conclude that $\Delta P_{1}^{(1)}=0$. Note that our 
linear theory is only valid up to $O\left(\Gamma^{2}\right)$ and thus we have shown that $\Delta P_{1}^{(1)}=0$ up to $O\left(\Gamma^{2}\right)$. By analogy with the analysis of $\S 3$ we might conjecture that $\Delta P_{1}^{(1)}$ is identically zero to all order in $\Gamma$ for two reasons. Firstly, the first perturbation to the pressure difference across the meniscus was found in $\S 3$ to be $O\left(\mathrm{Ca}^{2 / 3}\right)$ rather than $O\left(\mathrm{Ca}^{1 / 3}\right)$. Secondly, of all the numerical constants in (4.28) the right-hand side of (4.35) depends only on $B_{1}$, which is not invariant under translation in $\zeta$. When the full nonlinear wall law (2.5) is used, it can be shown that $\Delta P_{1}^{(1)}$ is indeed identically zero at all orders in $\Gamma$. We do not include the lengthy details here since we only consider terms up to $O(\Gamma)$.

Having established that $\Delta P_{1}^{(1)} \equiv 0$, we are still unable to determine the first nonzero perturbation to the pressure drop, i.e. $\Delta P_{1}^{(2)}$, from the information obtained thus far. The reason for this is that the contact angle for the meniscus is not, in contrast with $\S 3$, zero to leading order. This means that the $O\left(C a^{2 / 3}\right)$ coefficient in $\tan \theta_{c}$ is required to determine $\Delta P_{1}^{(2)} \dagger \dagger$ Thus we must go up to next order.

\subsection{Second-order correction}

\section{Intermediate region}

Substituting (4.8) into equations (4.6) and considering terms of $O\left(\mathrm{Ca}^{2 / 3}\right)$ we obtain two second-order o.d.e.s for $\tilde{w}^{(2)}(\xi)$ : one in $\xi<0$, and in $\xi>0$ that also involves the unknown $\Delta P_{1}^{(2)}$. As detailed in $\S 4.6$, given $\Delta P_{1}^{(2)}$ we can deduce a relation between $\tilde{w}_{\xi}^{(2)}(0-)$ and $\tilde{w}^{(2)}(0-)$, and a second relation between $\tilde{w}_{\xi}^{(2)}(0+)$ and $\tilde{w}^{(2)}(0+)$. These relationships are

$$
\begin{aligned}
& -\frac{1}{2}\left(2 W_{0} \tilde{c}_{1}+\lambda^{2}\right)+\frac{1}{2}(1+\bar{\alpha})\left(2 W_{1} \tilde{w}_{\xi}^{(2)}(0-)+b_{1}^{2}\right)=O(\Gamma), \\
& c_{1}\left[W_{0}+1-\Delta P_{1}^{(0)}\right]+\frac{1}{2} \lambda^{2}-W_{1} \tilde{w}_{\xi}^{(2)}(0+)-\frac{1}{2} b_{1}^{2}=\Delta P_{1}^{(2)} \Phi_{1} .
\end{aligned}
$$

(Note that in order to compute the leading-order solutions for the pressure drop across the bolus it is unnecessary to determine exactly the $O(\Gamma)$ correction in $(4.37 a)$.

\section{Inner region}

In order to smooth out discontinuities in the free-surface curvature, we must again consider the expansions for $w$ and $h$ in the inner region between the meniscus and the trailing film. Proceeding to higher order in the expansion we obtain equations for $W^{(3)}$ and $H^{(1)}$ :

$$
\begin{array}{r}
\left(1-\Gamma W_{0}\right) K_{\zeta}^{(0)} 3 H^{(0)^{2}} H^{(1)}+\left(1-\Gamma W_{0}\right) \mathrm{K}_{\zeta}^{(1)} H^{(0)^{3}}-\Gamma W^{(1)} \mathrm{K}_{\zeta}^{(0)} H^{(0)^{3}} \\
-\frac{\alpha}{\beta_{1}}\left(1-\Gamma W_{0}\right)^{3} H^{(1)}+\frac{\alpha}{\beta_{1}} \Gamma\left(1-\Gamma W_{0}\right)^{2} W^{(1)} H^{(0)}=\text { const. } \\
-(1-\Gamma)\left(\lambda+W_{1} \zeta\right)+(1+\bar{\alpha}) W_{\zeta \zeta}^{(3)}=-\delta H_{\zeta \zeta}^{(1)}+\delta \Gamma W_{1} H^{(0)}\left[\bar{\alpha} \delta W_{\zeta \zeta \zeta}^{(2)}+\delta H_{\zeta \zeta \zeta}^{(0)}\right],
\end{array}
$$

where $\mathrm{K}_{\zeta}^{(0)}=\bar{\alpha} W_{\zeta \zeta \zeta}^{(2)}+\delta H_{\zeta \zeta \zeta}^{(0)}$ and $\mathrm{K}_{\zeta}^{(1)}=\bar{\alpha} W_{\zeta \zeta \zeta}^{(3)}+\delta H_{\zeta \zeta \zeta}^{(1)}$. As before $(\S 4.3)$, these can be combined into a single o.d.e. for $H^{(1)}$ whose solution can, in principle, be found numerically, although this turns out to be unnecessary.

$\uparrow$ For a simple minded explanation, consider the expansion

$$
\cos \left(\theta_{0}+\varepsilon \theta_{1}+\varepsilon^{2} \theta_{2}+\cdots\right) \sim \cos \theta_{0}-\varepsilon \theta_{1} \sin \theta_{0}-\varepsilon^{2}\left(\theta_{2} \sin \theta_{0}+\frac{1}{2} \theta_{1}^{2} \cos \theta_{0}\right) .
$$

Notice that for $\theta_{0} \equiv 0$, the first perturbation is $O\left(\varepsilon^{2}\right)$ and does not depend on $\theta_{2}$. For $\theta_{0} \neq 0$, the first perturbation is $O(\varepsilon)$, and to find the coefficient of $\varepsilon^{2}$, we need to know $\theta_{2}$. 
Matching

By matching the expression for the wall displacement, $W$, in the inner region with the intermediate solution, $\tilde{w}$, in and behind the bolus it is seen that in general $W^{(3)}$ behaves asymptotically as a cubic function of $\zeta$ as $\zeta \rightarrow \pm \infty$. Matching the film thickness, $H$, as $\zeta \rightarrow-\infty$ with the intermediate solution, we find that $H^{(1)}$ is linear at $\zeta=-\infty$. Finally, matching the free-surface position in the inner region as $\zeta \rightarrow+\infty$ with the outer capillary static solution we see that $H^{(1)}$ is cubic at $+\infty$. For the purpose of finding $\Delta P_{1}^{(2)}$ we need only consider the linear term in each case:

$$
\begin{aligned}
& H^{(1)} \sim \cdot \zeta^{3}+\cdot \zeta^{2}+D_{1} \zeta+\cdots, \quad W^{(3)} \sim \cdot \zeta^{3}+\cdot \zeta^{2}+d_{1} \zeta+\cdots \quad \text { as } \zeta \rightarrow+\infty, \\
& \left.H^{(1)} \sim \tilde{D}_{1} \zeta+\cdots, \quad W^{(3)} \sim \cdot \zeta^{3}+\cdot \zeta^{2}+\tilde{d}_{1} \zeta+\cdots \quad \text { as } \rightarrow-\infty,\right\}
\end{aligned}
$$

where the expression for $\tilde{D}_{1}$ is

$$
\tilde{D}_{1}=\left(1-\Gamma W_{0}\right) \tilde{h}_{\xi}^{(0)}(0-)=\frac{\Gamma W_{1}}{1-\Gamma W_{0}},
$$

and the expressions for $d_{1}$ and $\tilde{d}_{1}$ are

$$
d_{1}=\tilde{w}_{\xi}^{(2)}(0+), \quad \tilde{d}_{1}=\tilde{w}_{\xi}^{(2)}(0-),
$$

respectively. We can determine $D_{1}$ by numerically integrating the o.d.e. for $H_{1}$, although for the purpose of finding the leading-order correction to the pressure drop across the bolus this is not necessary.

Furthermore, by integrating (4.38b) with respect to $\zeta$ and using the expressions for $W^{(3)}$ and $H^{(1)}$ at $\pm \infty(4.39)$ we can deduce the following condition:

$$
(1+\bar{\alpha})\left(d_{1}-\tilde{d}_{1}\right)+\delta\left(D_{1}-\tilde{D}_{1}\right)-\delta^{2} W_{1} \frac{\Gamma}{1+\bar{\alpha}} F_{1}=0 .
$$

Finally, matching the outer capillary static solution to the inner solution, as in $\S 4.5$ gives

$$
R_{z}^{(2)}(0)=-\frac{\alpha^{2} \beta_{1}^{2}}{\sqrt{\hat{T}}}\left(\bar{\alpha} d_{1}+\delta D_{1}\right)
$$

and, from (3.5), the first non-zero perturbation to the pressure is given by

$$
\begin{aligned}
\Delta P_{1}^{(2)}= & \frac{2 \Gamma}{\left(1-\Gamma W_{0}\right) \sqrt{1+\bar{\alpha} \Gamma W_{1}^{2}}} \\
& \times\left(\frac{\bar{\alpha} c_{1}+\delta c_{1}}{\bar{\alpha}\left(1-\Gamma W_{0}\right)}-\frac{\left(1-2 \bar{\alpha} \Gamma W_{1}^{2}\right)\left(\bar{\alpha} b_{1}+\delta B_{1}\right)^{2}}{2 \bar{\alpha}\left(1+\bar{\alpha} \Gamma W_{1}^{2}\right)^{2}}-\frac{W_{1}\left(\bar{\alpha} d_{1}+\delta D_{1}\right)}{\left(1+\bar{\alpha} \Gamma W_{1}^{2}\right)}\right) .
\end{aligned}
$$

From $(4.31 c),(4.37)$ and (4.42) we can determine the constants $c_{1}, \tilde{c}_{1}, d_{1}, \tilde{d}_{1}$ in (4.44) in terms of $\Delta P_{1}^{(2)}$ and $D_{1}$. When these expressions are substituted into (4.44) we get

$$
\Delta P_{1}^{(2)}=\frac{1}{\bar{\alpha} \alpha^{2} \beta_{1}^{2}} \Gamma\left[A_{1} \Phi_{3}+F_{1} \Phi_{4}\right]+O\left(\Gamma^{2}\right),
$$

where $F_{1}=A_{1} C_{1}-\frac{1}{2} B_{1}^{2}$ and

$$
\left.\begin{array}{rl}
\Phi_{3} & =2 \frac{1}{(1+\bar{\alpha})^{1 / 3}}\left(\frac{\bar{\alpha} W_{0}}{1+\bar{\alpha}-\bar{\alpha} W_{0}}+O(\Gamma)\right)=2 \frac{1}{(1+\bar{\alpha})^{1 / 3}}((\sqrt{1+\bar{\alpha}}-1)+O(\Gamma)), \\
\Phi_{4} & =2 \frac{1}{(1+\bar{\alpha})^{1 / 3}}(1+O(\Gamma)) .
\end{array}\right\}
$$


(Note that the leading-order (in $\Gamma$ ) expression for the pressure drop across the rear meniscus is independent of $D_{1}$, so that we did not need to determine this constant numerically).

The pressure drop across the rear meniscus, $\Delta P_{1}$, is given by

$$
\Delta P_{1} \sim \Delta P_{1}^{(0)}+C a^{2 / 3}\left[A_{1} \Phi_{3}+F_{1} \Phi_{4}\right]+\cdots,
$$

where $\Delta P_{1}^{(0)}$ is given in (4.20).

This completes the analysis for the propagation of a semi-infinite bubble into a liquid-filled tube: the configuration analysed numerically by Gaver et al. (1996). The pressure drop across the meniscus is related to the propagation speed by (4.47), and the trailing film thickness is given by (4.33). The first perturbation to the pressure drop across the meniscus takes the form of a linear combination of the two invariants of (4.28). This contrasts with $\S 3$, where only the invariant $F_{1}$ was found to affect the pressure drop. A second contrast is that here we need only integrate the o.d.e. (4.27) once to determine $A_{1}$ and $F_{1}$; in the previous section the constants were found to depend on the elastic properties of the tube wall, so that the o.d.e. (3.15) had to be integrated anew for each value of the flexibility parameter $G_{1}$.

\subsection{The front meniscus}

Now the preceeding analysis is repeated for the front meniscus. Most of the results derived thus far apply to the front meniscus with minor modifications, so we omit many of the details.

The leading-order problem, and hence the definitions of $\tilde{w}^{(0)}, W^{(0)}$ and $W^{(1)}$ are identical to those in $\S 4.2$. Hence the solution for the leading-order pressure drop across the front meniscus, $\Delta P_{2}^{(0)}$, is equal to $\Delta P_{1}^{(0)}$. Likewise, the analysis determining the first- and second-order corrections to the pressure drop across the rear meniscus, detailed in $\S 4.6$ and 4.7 , is followed closely, here setting

$$
h_{2}=\beta_{2} C a^{2 / 3} \text {, }
$$

and expanding in the form

$$
\tilde{w}=\tilde{w}^{(0)}+\frac{\alpha \beta_{2} C a^{1 / 3}}{\sqrt{\hat{T}}} \tilde{w}^{(1)}+\cdots, \text { etc. }
$$

Of course, in the inner region, we now have to solve the 'forward' Landau-Levich problem

$$
\left(H^{(0)}\right)^{3} H_{\zeta \zeta \zeta}^{(0)}=H^{(0)}-1, \quad H^{(0)} \rightarrow 1 \quad \text { as } \zeta \rightarrow+\infty .
$$

which, as noted in $\S 3$, has a one-parameter family of solutions, whose general asymptotic behaviour is

$$
H^{(0)} \sim \frac{1}{2} A_{2} \zeta^{2}+B_{2} \zeta+C_{2} \text { as } \zeta \rightarrow-\infty .
$$

The function relationship between the invariants $F_{2}=A_{2} C_{2}-\frac{1}{2} B_{2}^{2}$ and $A_{2}$ is the same as for the classical stiff tube problem (Wong et al. 1995a,b) and is shown in the $G_{2}=0$ curve in figure 3(a). Also the precursor thickness $h_{2}$ is related to $A_{2}$ just as $h_{1}$ is to $A_{1}$ in (4.33).

The only difference between the front and rear menisci is that the gas pressure is $P_{2}$, not $P_{1}$ (they differ by $\Delta P \sim O\left(C a^{2 / 3}\right)$ ). This causes the wall displacement ahead of the bolus to be different from that behind:

$$
\tilde{w} \rightarrow \frac{\beta_{2} C a^{2 / 3}+\Delta P}{1-\Gamma} \text { as } \zeta \rightarrow \infty .
$$




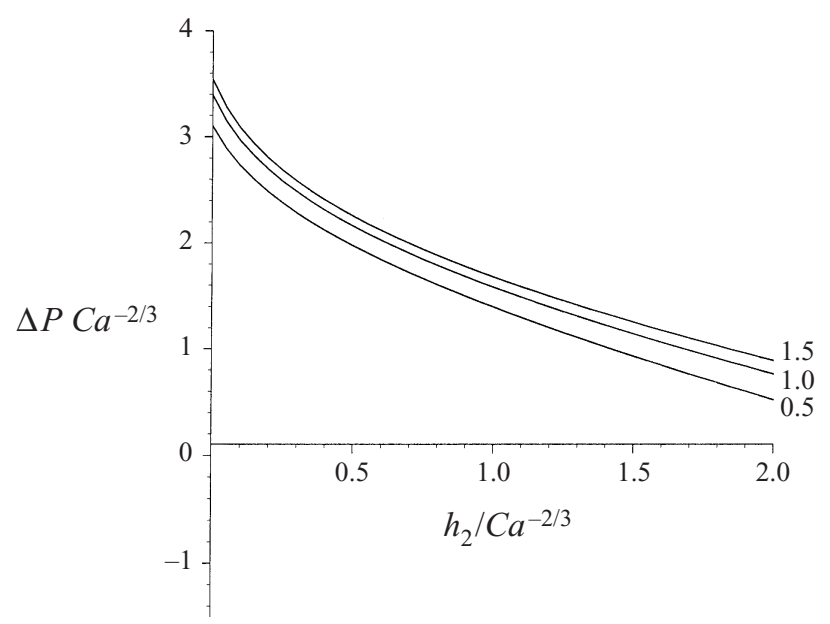

FIGURE 8. Dimensionless pressure drop across the bolus versus dimensionless precursor thickness for various values of the dimensionless longitudinal wall tension, $T=T_{l} / \sigma$.

The equation for $\Delta P_{2}$ is

$$
\Delta P_{2} \sim \Delta P_{2}^{(0)}+C a^{2 / 3}\left[A_{2} \Phi_{3}+F_{2} \Phi_{4}\right]+\cdots,
$$

where $\Phi_{3}$ and $\Phi_{4}$ are given in (4.46). Given the precursor film thickness $h_{2}$, the constant $A_{2}$ is given by

$$
A_{2}=\frac{h_{2}}{C a^{2 / 3}} \alpha^{2}\left(1-\Gamma W_{0}\right)\left[-(1+\bar{\alpha})\left(1-\Delta P_{1}^{(0)}+W_{0}\right)+W_{0}(1-\Gamma)\right] .
$$

From (4.47) and (4.53), the total pressure drop across the bolus is related to its propagation speed by

$$
\Delta P=\Delta P_{1}-\Delta P_{2}=C a^{2 / 3}\left(\Phi_{3}\left(A_{1}-A_{2}\right)+\Phi_{4}\left(F_{1}-F_{2}\right)\right),
$$

and, at leading order, the expression for the pressure drop across the bolus is

$$
\begin{aligned}
\Delta P & =C a^{2 / 3} \frac{2}{(1+\bar{\alpha})^{1 / 3}}\left\{(\sqrt{1+\bar{\alpha}}-1)\left(A_{1}-A_{2}\right)+\left(F_{1}-F_{2}\right)\right\} \\
& =C a^{2 / 3} \frac{2 T^{1 / 3}}{(1+T)^{1 / 3}}\left\{\left(\frac{\sqrt{1+T}}{\sqrt{T}}-1\right)\left(A_{1}-A_{2}\right)+\left(F_{1}-F_{2}\right)\right\} .
\end{aligned}
$$

For fixed $h_{2}$ and $\mathrm{Ca}$, the pressure drop across the bolus increases as $T$ increases. However, for fixed $C a$ and $T$ the pressure drop across the bolus decreases as the precursor film thickness increases. This can be clearly seen in figure 8 where $\triangle P C a^{-2 / 3}$ is plotted against $h_{2} / \mathrm{Ca}^{2 / 3}$ for various values of $T$.

The leading-order trailing film thickness is given by

$$
h_{1}=\beta_{1} C a^{2 / 3}=A_{1} C a^{2 / 3}(1+\bar{\alpha})^{1 / 6}=A_{1} C a^{2 / 3} \frac{(1+T)^{1 / 6}}{T^{1 / 6}},
$$

so that increasing the wall tension $T$ results in a decrease in the trailing film thickness $h_{1} /\left(C a^{2 / 3}\right)$, as seen in figure 9 .

We can identify the critical capillary number and corresponding pressure drop for the trailing film thickness to exceed the precursor film thickness, so that the tube 


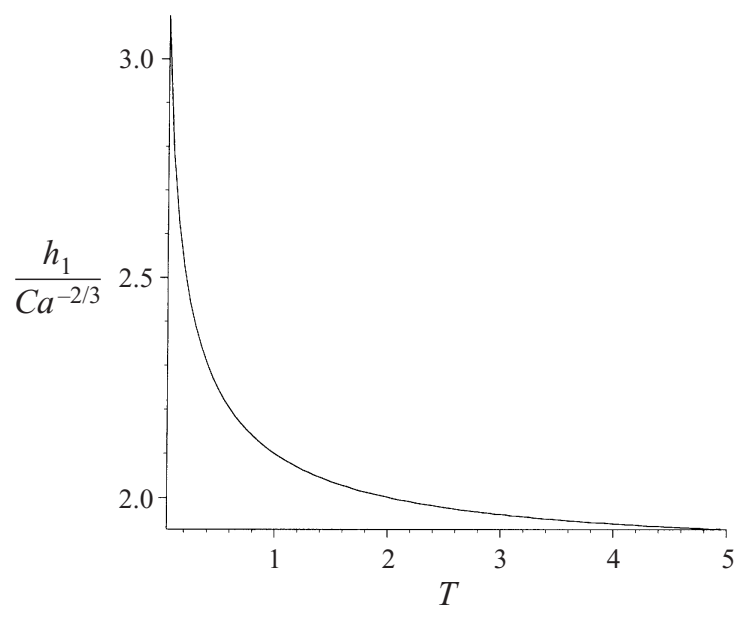

FIGURE 9. Dimensionless trailing film thickness versus the longitudinal wall tension.
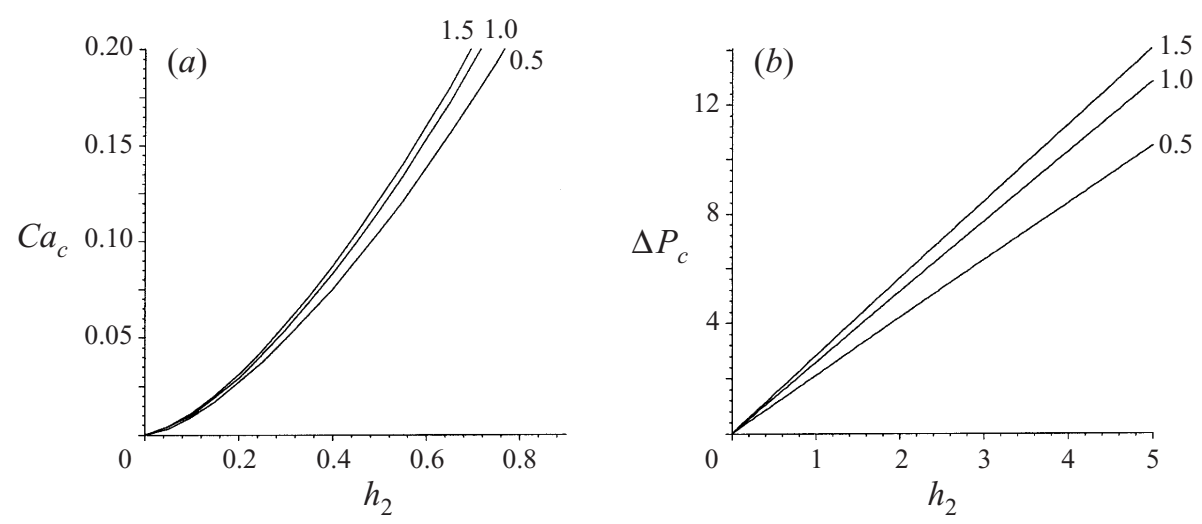

FiguRE 10. (a) Critical capillary number and (b) critical dimensionless pressure drop versus dimensionless precursor film thickness for various values of the longitudinal wall tension.

must eventually reopen. These take the forms

$$
\begin{gathered}
C a_{c}=1.94 h_{2}^{3 / 2} \frac{1}{(1+\bar{\alpha})^{1 / 4}}=1.94 h_{2}^{3 / 2} \frac{T^{1 / 4}}{(1+T)^{1 / 4}}, \\
\Delta P_{c}=3.63 h_{2} \frac{1}{\sqrt{1+\bar{\alpha}}}=3.63 h_{2} \frac{\sqrt{T}}{\sqrt{1+T}} .
\end{gathered}
$$

For fixed precursor film thickness, $h_{2}$, the reopening capillary number and pressure drop both increase as $T$ increases (see figure 10). For fixed tension, both the reopening capillary number and the pressure drop increase as the precursor film thickness increases.

In figure 11, we plot capillary number versus pressure drop across the bolus for a range of values of the wall tension. As the wall tension increases, the speed of the bolus, for a given pressure drop, decreases. Hence the bolus propagation speed decreases as the wall tension increases.

Finally, in figure 12 we plot the air-liquid and liquid-wall positions for various values of the wall compliance and capillary number. In figure 12(a) the wall compliance 


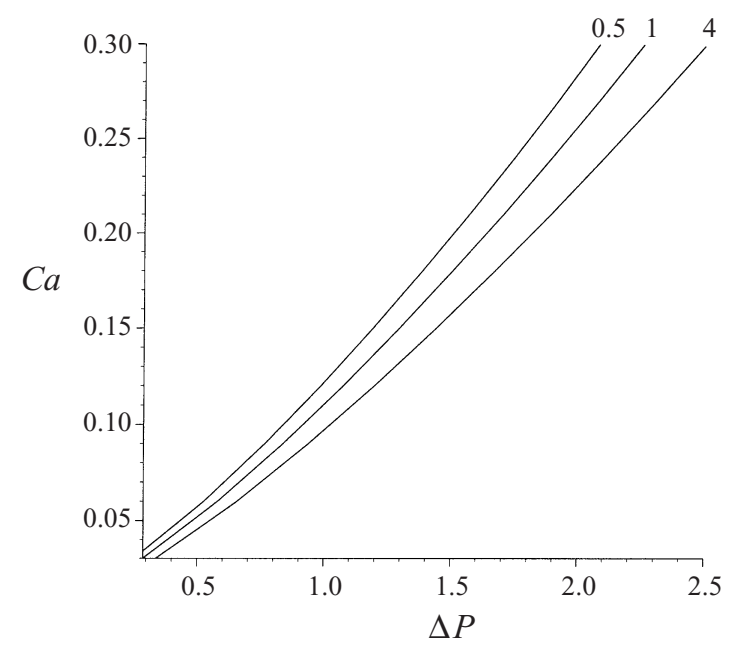

FIGURE 11. Capillary number versus dimensionless pressure drop for various values of the longitudinal wall tension $\left(h_{2}=0.1\right)$.
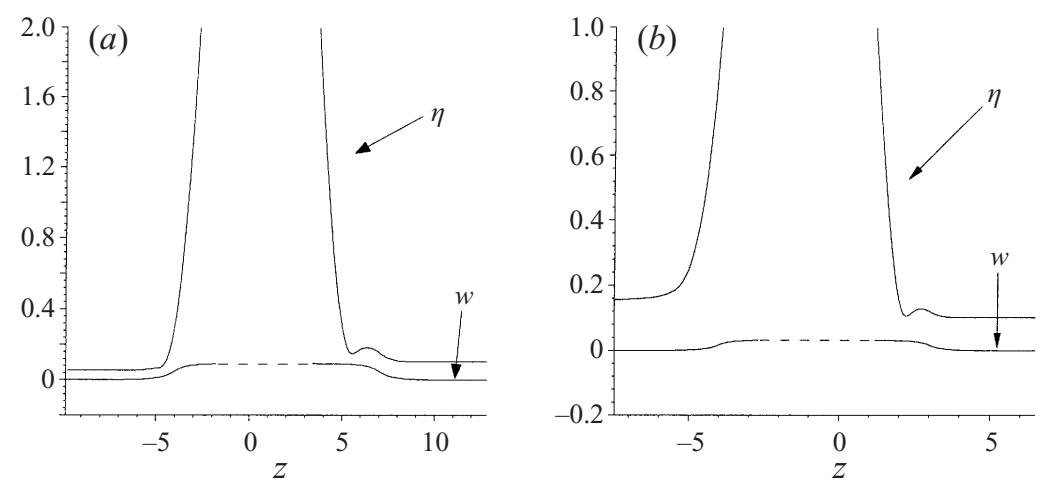

FIGURE 12. Solution for the film height and wall displacement ahead of and behind the bolus. (a) $C a=0.02, h_{2}=0.1, \Gamma=0.1, T=4$; (b) $C a=0.1, h_{2}=0.1, \Gamma=0.03, T=4$.

$\Gamma=0.1$ and capillary number $C a=0.02$. The value of the precursor film thickness is $h_{2}=0.1$ and we choose a value for the longitudinal wall tension of $T=4$. The trailing film thickness is less than the precursor film thickness so that the tube will not reopen. Note that, due to the presence of longitudinal wall tension, there is no longer a region of rapid change in the wall displacement ahead of the bolus (compare with figure 6). In figure $12(b)$ the wall compliance $\Gamma=0.03$ and capillary number $C a=0.1$. In this case the trailing film thickness exceeds the precursor film thickness so that the tube will eventually reopen.

\section{Conclusions}

We have used low capillary number asymptotics to model the propagation of a liquid bolus along a liquid-lined flexible tube under an applied pressure difference. We obtained leading-order expressions for the pressure difference across the bolus and the thickness of the film it leaves behind, as functions of the capillary number, the thickness of the liquid lining ahead of the bolus and the elastic properties of the 
tube wall. From these expressions we deduced the critical pressure drop which must be applied across a bolus if the thickness of the film it leaves behind is to exceed that of the film ahead of it. This was identified as the critical pressure drop for eventual reopening of the tube.

Two different scaling choices have been considered. In $\S 3$ we employed a limit in which the longitudinal wall tension was very small. We also assumed that the dimensionless wall compliance was the same order as the trailing film thickness so that the stiff tube analyses of previous papers could be followed closely. As in those papers, surface tension and viscous drag were found to balance in transition regions joining the capillary static menisci to the uniform films on either side of the bolus. We found that the film thickness in these regions satisfies a nonlinear, third-order differential equation (3.15), that generalizes the celebrated Landau-Levich equation. Asymptotic expressions for the trailing film thickness and pressure drop were found by matching numerical solutions of (3.15) with a meniscus on one side and a uniform film on the other. Our conclusions were:

(i) the trailing film thickness is an increasing function of the capillary number (like $C \mathrm{a}^{2 / 3}$ ), and of the wall compliance;

(ii) the pressure drop across the bolus is an increasing function of the capillary number (like $C a^{2 / 3}$ ), and a decreasing function of the wall compliance and the precursor film thickness;

and hence

(iii) increasing wall compliance and precursor film thickness makes the tube easier to reopen.

The derivation and solution of (3.15) were relatively straightforward, but the price of this is some severe limitations on the validity and utility of the theory in $\S 3$. These include the following:

(i) the theory assumes vanishingly small wall compliance, namely $\Gamma \sim O\left(\mathrm{Ca}^{2 / 3}\right)$, and that the longitudinal wall tension is very small;

(ii) the differential equation (3.15) must be integrated for each different value of the flexibility parameter $G_{1}$;

(iii) the solutions (3.22) and (3.29) for $h_{1}^{*}$ and $\Delta P$ take an awkward, coupled, implicit form, since $G_{1}$ depends on $h_{1}^{*}$.

To remedy these weaknesses, in $\S 4$ we allowed for greater longitudinal tension and wall compliance. In particular we made the (more realistic) assumption that the compliance parameter is not correlated with $\mathrm{Ca}$ as $\mathrm{Ca} \rightarrow 0$. This made the derivation much more complicated as the tube is no longer cylindrical at leading order. Nevertheless a lubrication analysis of the transition regions analogous to that employed in $\S 3$ was successfully carried out and, by appropriate rescaling, the film thickness was found to satisfy the Landau-Levich equation. As in $\S 3$, asymptotic matching of the numerical solution gave expressions for the trailing film thickness and pressure drop.

In contrast with $\S 3$, it was necessary to proceed to higher order in the capillary number in the inner region to determine the first non-zero perturbation to the pressure drop. Fortunately, it was not necessary to solve the higher-order problem since the required information was deduced from its solvability conditions. Despite the greater complexity of the derivations in $\S 4$, the results thereof are in many ways simpler than those in $\S 3$. For example the elastic parameters were scaled out of the o.d.e. (4.27) for the film thickness in the transition region, which therefore need only be solved once. Also, the dependence of the trailing film thickness and pressure drop on the capillary number and elastic parameters was explicit and decoupled. 
Our conclusions of $\S 4$ were:

(i) the trailing film thickness is an increasing function of the capillary number (like $\mathrm{Ca}^{2 / 3}$ ) and a decreasing function of the wall tension, $T$;

(ii) the pressure drop across the bolus is an increasing function of the capillary number (like $C a^{2 / 3}$ ), and of the wall tension, $T$;

and hence

(iii) increasing wall tension makes the tube harder to reopen.

Note that these results are consistent with the results of $\S 3$, since increasing $T$ is equivalent to decreasing wall compliance.

In the weak tension limit, we found that the critical pressure drop which must be applied across a bolus if the thickness of the film it leaves behind is to exceed that of the film ahead of it decreases as the wall compliance increases. Thus in general a more compliant tube is easier to reopen. This mirrors the result (Halpern \& Grotberg 1992) that a more compliant tube is more susceptible to closure. In the tension dominated limit, we found that the critical reopening pressure drop is an increasing function of $T$ so that increasing wall tension makes the tube harder to reopen. We also found (not surprisingly) that, in both cases, increasing the thickness of the tube's liquid lining, which clearly makes it more likely to close, also makes it harder to reopen.

The length of the bolus plays no part in our analysis. This is a result of our implicit assumption that the bolus length, $L_{b}$, is of the same order as the tube radius, $a$. If the bolus is much longer than the tube radius $\left(L_{b} / a \gtrsim O\left(\mathrm{Ca}^{-1 / 3}\right)\right)$, viscous drag contributes significantly to the pressure drop across the bolus. On the other hand if the bolus becomes very short, then the interaction between the two menisci can no longer be neglected: they can be coupled through the tube wall response (for $\left.L_{b} / a \lesssim O(\sqrt{\hat{T}})\right)$ and through fluid mechanics in the bolus (for $\left(L_{b} / a \lesssim O\left(C a^{-1 / 3}\right)\right.$ ).

In $\S 4$ we found that in general the first perturbation to the pressure drop across a moving meniscus in a flexible tube may be $O\left(\mathrm{Ca}^{1 / 3}\right)$, rather than $O\left(\mathrm{Ca}^{2 / 3}\right)$ as in the classical theory for a stiff tube. This appears to contradict the observation that the viscous drag on the meniscus is proportional to $\mathrm{Ca}^{2 / 3}$ (Ratulowski \& Chang 1989; Wong et al. 1995a, b). Notice that the proof that (for our particular wall model) the coefficient of $\mathrm{Ca}^{1 / 3}$ is identically zero rests on the property that the wall law (2.8) admits first integrals. This property reflects the fact that our wall law describes a conservative elastic response, i.e. that it introduces no new dissipation to the problem. We conjecture that any other conservative wall law would result in the same dependence on the capillary number. If, however, we allow non-conservative behaviour of the tube wall, for example active response (as in peristalsis) or visco-elastic damping, then a different dependence on the capillary number must be anticipated in general (Gaver et al. 1996).

\subsection{Application to the lung}

Here we estimate the dimensionless parameters of the problem based on the properties and dimensions of small airways which are prone to developing a fluid plug. The values are those given in Halpern \& Grotberg (1992). The thickness of the liquid lining is approximately $10 \%$ of the tube radius and its viscosity is about the same as water, $\mu=0.01 \mathrm{~g} \mathrm{~cm}^{-1} \mathrm{~s}^{-1}$, since the cells lining the airway past generation 15 lack mucus secreting cells. The tube radius, based on Olson, Dart \& Filley (1970) is $a=2.5 \times 10^{-2} \mathrm{~cm}$. The parameters characterizing the wall are Young's modulus $E=6 \times 10^{4} \mathrm{dyn} \mathrm{cm}^{-2}$, thickness of the wall $d=2.5 \times 10^{-3} \mathrm{~cm}$, Poisson ratio $v=0.5$ and longitudinal wall tension $T_{l}=25 \mathrm{dyn} \mathrm{cm}^{-1}$ (based on a pleural pressure of $-5 \mathrm{~cm} \mathrm{H}_{2} \mathrm{O}$ ) (Halpern \& Grotberg 1992). We also take the surface tension $\sigma$ to 
be $\sim 20 \mathrm{dyn}^{-2}$. Using these values, the dimensionless compliance $\Gamma \sim 0.1$ and the dimensionless longitudinal wall tension is $T \sim 1.25$. Hence, $T$ lies in the range $\Gamma \ll T \ll \Gamma^{-1}$ and so our analysis for the tension dominated case, $\S 4$, is most applicable to the lung.

This work was supported by NASA grant NAG3-2196, NSF grant CTS-9412523 and NIH grant NIH-HL41126. The authors also wish to acknowledge the helpful advice of Professor M. Miksis, Dr H. Wong and Dr D. Halpern.

\section{REFERENCES}

AtABEK, H. B. \& LEW, H. S. 1966 Wave propagation through a viscous incompressible fluid contained in an initially stressed elastic tube. Biophys. J. 6, 481-503.

Bretherton, F. P. 1961 The motion of long bubbles in tubes. J. Fluid Mech. 10, 166-188.

Gaver III, D. P., Halpern, D. M., Jensen, O. E. \& Grotberg, J. B. 1996 The steady motion of a semi-infinite bubble through a flexible-walled channel. J. Fluid Mech. 319, 25-65.

Goren, S. L. 1962 The instability of an annular thread of fluid. J. Fluid Mech. 12, 309-319.

Halpern, D. \& Grotberg, J. B. 1992 Fluid-elastic instabilities of liquid-lined flexible tubes. J. Fluid Mech. 244, 615-632.

Halpern, D. \& Grotberg, J. B. 1993 Surfactant effects on fluid-elastic instabilities of liquid-lined flexible tubes: a model of airway closure. J. Biomech. Engng 115, 271-277.

Hammond, P. S. 1983 Nonlinear adjustment of a thin annular film of viscous fluid surrounding a thread of another within a circular pipe. J. Fluid Mech. 137, 363-384.

Hughes, J. M. B., Rosenzweig, D. Y. \& Kivitz, P. B. 1970 Site of airway closure in excised dog lungs: histologic demonstration. J. Appl. Physiol. 29, 340-344.

Johnson, M., Kamm, R. D., Ho, L. W., Shapiro, A. \& Pedley, T. J. 1991 The nonlinear growth of surface-tension-driven instabilities of a thin annular film. J. Fluid Mech. 233, 141-156.

Kamm, R. D. \& Schroter, R. C. 1989 Is airway closure caused by a thin liquid instability? Respir. Physiol. 75, 141-156.

Landau, L. D. \& Levich, V. G. 1942 Dragging of a liquid by a moving plate. Acta Physicochim. URSS 17, 42-54.

Macklem, P. T., Proctor, D. F. \& Hogg, J. C. 1970 The stability of peripheral airways. Respir. Physiol. 8, 191-203.

Olson, D. E., Dart, G. A. \& Filley, G. F. 1970 Pressure drop and fluid flow regime of air inspired into the human lung. J. Appl. Physiol. 28, 482-494.

Otis, D. R. Jr., Johnson, M., Pedley, T. J. \& Kamm, R. D. 1993 Role of pulmonary surfactant in airway closure: a computational study. J. Appl. Physiol. 75, 1323-1333.

Park, C.-W. \& Homsy, G. M. 1984 Two-phase displacement in hele shaw cells: theory. J. Fluid Mech. 139, 291-308.

Prisk, G. K., Guy. H. J., Elliott, A. R., Paiva, M. \& West, J. B. 1995 Ventilatory inhomogeneity determined from multiple-breath washouts during sustained microgravity on Spacelab SLS-1. J. Appl. Physiol. 78, 597-607.

Ratulowski, J. \& Chang, H.-C. 1989 Transport of gas bubbles in capillaries. Phys. Fluids A 1, $1642-1655$.

RaYleigh, LoRd 1879 On the Instability of Cylindrical Fluid Surfaces. Cambridge University Press.

RAYLEIGH, LORD 1892 On the instability of a cylinder of viscous liquid under capillary force. Phil. Mag. 34, 145.

RuschaK, K. J. \& Scriven, L. E. 1977 Developing flow on a vertical wall. J. Fluid Mech. 81, 305-316.

Schwartz, L. W., Princen, H. M. \& Kiss, A. D. 1986 On the motion of bubbles in capillary tubes. J. Fluid Mech. 172, 259-275.

Van Dyke, M. 1964 Perturbation Methods in Fluid Mechanics. Academic.

WiLson, S. D. R. 1982 The drag-out problem in film coating theory. J. Engng Maths 16, 209-216.

WONG, H., RAdKe, C. J. \& MoRRIS, S. $1995 a$ The motion of long bubbles in polygonal capillaries. Part 1. Thin films. J. Fluid Mech. 292, 71-94.

Wong, H., RADKe, C. J. \& MorRIS, S. $1995 b$ The motion of long bubbles in polygonal capillaries. Part 2. Drag, fluid pressure and fluid flow. J. Fluid Mech. 292, 95-110. 